Review Article

\title{
Atom-bond connectivity index of graphs: a review over extremal results and bounds
}

\author{
Akbar Ali ${ }^{1,2}$, Kinkar Ch. Das ${ }^{3}$, Darko Dimitrov ${ }^{4}$, Boris Furtula ${ }^{5, *}$ \\ ${ }^{1}$ Department of Mathematics, Faculty of Science, University of Hail, Hail, Saudi Arabia \\ ${ }^{2}$ Knowledge Unit of Science, University of Management and Technology, Sialkot, Pakistan \\ ${ }^{3}$ Department of Mathematics, Sungkyunkwan University, Suwon 16419, Republic of Korea \\ ${ }^{4}$ Faculty of Information Studies, Novo mesto, Slovenia \\ ${ }^{5}$ Faculty of Science, University of Kragujevac, Kragujevac, Serbia
}

(Received: 7 December 2020. Received in revised form: 26 January 2021. Accepted: 26 January 2021. Published online: 29 March 2021.)

(C) 2021 the authors. This is an open access article under the CC BY (International 4.0) license (www.creativecommons.org/licenses/by/4.0/).

\begin{abstract}
The atom-bond connectivity $(A B C)$ index was introduced in the last quarter of the 1990s to improve the prediction power of the Randić index. Later on, in 2008, the factor $\sqrt{2}$ was dropped from the original definition of the $A B C$ index, and some additional chemical applications of this index were reported, which resulted in considerable interest in studying the mathematical properties of the $A B C$ index. There are more than a hundred papers devoted to the mathematical aspects of this graph invariant. The primary purpose of this review is to gather the existing bounds and extremal results concerning the $A B C$ index.
\end{abstract}

Keywords: chemical graph theory; graph invariant; topological index; atom-bond connectivity index; $A B C$ index.

2020 Mathematics Subject Classification: 05C09, 05C35, 05 C92.

\section{Introduction}

Throughout this survey, by the term "graph", we mean a finite graph containing neither any loop nor multiple edges. The edge set and vertex set of a graph $G$ are denoted by $E(G)$ and $V(G)$, respectively. Degree of an arbitrary vertex $w \in V(G)$ is denoted by $d_{w}$. Denote by $u v$ the edge connecting the vertices $u, v \in V(G)$. Further notation, as well as terminology, are given in section 2, and those that are not defined here can be found in some relevant books, like [16, 17, 137].

A numerical quantity $I$ associated with a graph $G$ satisfying the equation $I(G)=I\left(G^{\prime}\right)$ for every graph $G^{\prime}$ isomorphic to $G$ is called a graph invariant. In chemical graph theory, the graph invariants having that are applied in the chemical investigations are known as the topological indices. A chemical compound can be viewed as a graph in which vertices represent atoms and edges represent bonds. Such graphs are known as chemical/molecular graphs.

In 1975, Randić [127] devised the following topological index, under the name "branching index", for predicting the physicochemical properties of certain chemical compounds:

$$
R(G)=\sum_{u v \in E(G)} \frac{1}{\sqrt{d_{u} d_{v}}} .
$$

Nowadays, the branching index is also called the connectivity index and the Randic index. According to Gutman [85], "Randić index is the most investigated, most often applied, and most popular among all topological indices. Hundreds of papers and a few books are devoted to this topological index". Details about the Randic index can be found in the surveys $[107,128,129]$, books $[86,103,105]$, and in the related references listed therein.

In 1998, Estrada et al. [73] proposed a modified version of the Randić index, under the name atom-bond connectivity $(A B C)$ index, within a study of molecular modeling. Initially, the $A B C$ index of a graph $G$ was defined [73] as:

$$
A B C(G)=\sqrt{2} \sum_{u v \in E(G)} \sqrt{\frac{d_{u}+d_{v}-2}{d_{u} d_{v}}},
$$

but later on, this index was very slightly redefined [71] by dropping the factor $\sqrt{2}$. In the remaining part of this paper, we also neglect the factor $\sqrt{2}$ from the original definition of the $A B C$ index.

The $A B C$ index was initially devised for predicting the heats of formation of alkanes [73]. Later, Estrada [71] examined whether this index can be used to predict some other physicochemical properties of molecules and found that it provides 
also a good model for the stability of linear and branched alkanes as well as for the strain energy of cycloalkanes. A critical re-examination of the $A B C$-prediction-ability was conducted in [89], and it was concluded that it may successfully be applied to predict the heats of formation of alkanes. Possible applications of the $A B C$ index was discussed also in several other papers, for example see $[6,48,76,126]$.

Although the $A B C$ index was introduced in 1998, it did not gain much attention from the mathematical community till 2008, when several promising chemical applications were reported in the paper [71]. After the appearance of the paper [71], studies on the mathematical aspects of the $A B C$ index were initiated in the paper [77]. This paper introduced the $A B C$ index to the mathematical community, which resulted in more than a hundred publications dealing with its mathematical properties. Such types of publications are still appearing at a considerable pace. Most of these articles are related to extremal results or bounds. Thereby, it is meaningful to have a review on this index covering extremal results and bounds of the $A B C$ index - which is the main motivation for writing this survey paper. To avoid a too long survey, the variants of the $A B C$ index will be not considered. An interested can find some extremal results of the variants and modifications of the $A B C$ index in $[63,115,135]$ and references cited therein.

The remaining part of the present paper is organized as follows. The notation and definitions that are used here are given in the next section. Section 3 comprises two subsections: the first one is devoted to the upper bounds of the $A B C$ index, while the second one is reserved for its lower bounds. Section 4 also consists of two subsections: the first one gives the extremal results for the maximum $A B C$ index and the second one is about the extremal results regarding the minimum $A B C$ index.

\section{Preliminaries}

Most of the well-known topological indices that depends on only vertex degrees of the considered graph, are the special cases of the following graph invariant [96,140]:

$$
B I D(G)=\sum_{u v \in E(G)} f\left(d_{u}, d_{v}\right)
$$

where $f$ is a non-negative real-valued symmetric function of $d_{u}$ and $d_{v}$. The topological indices of the form (1) are referred to as the bond incident degree indices [138], BID indices in short [7]. In Table 1, the f-functions of some well-known topological indices (that will be used in the next sections) are given.

Table 1: Some topological indices considered in the present review. It needs to be mentioned that the modified second Zagreb index $M_{2}^{*}$ coincides with the so-called first-order overall index [15,120].

\begin{tabular}{|l|l|l|}
\hline Function $f\left(d_{u}, d_{v}\right)$ & Eq. (1) corresponds to & Symbol \\
\hline$d_{u}+d_{v}$ & first Zagreb index [88,90] & $M_{1}$ \\
$2\left(d_{u}+d_{v}\right)^{-1}$ & harmonic index [74] & $H$ \\
$\left(d_{u}+d_{v}\right)^{-1 / 2}$ & sum-connectivity index [154] & $X$ \\
$d_{u} d_{v}$ & second Zagreb index [88] & $M_{2}$ \\
$\left(d_{u} d_{v}\right)^{-1 / 2}$ & Randić index [127] & $R$ \\
$\left(\max \left\{d_{u}, d_{v}\right\}\right)^{-1}$ & modified Randić index [70] & $R^{\prime}$ \\
$\left(d_{u} d_{v}\right)^{-1}$ & modified second Zagreb index [120] & $M_{2}^{*}$ \\
$\sqrt{\frac{d_{u}+d_{v}-2}{d_{u} d_{v}}}$ & atom-bond connectivity index [73] & $A B C$ \\
$2 \sqrt{d_{u} d_{v}}\left(d_{u}+d_{v}\right)^{-1}$ & (first) geometric-arithmetic index [139] & $G A$ \\
$\frac{d_{u}}{d_{v}}+\frac{d_{v}}{d_{u}}$ & symmetric division deg index [140] & $S D D$ \\
$\left(\frac{d_{u} d_{v}}{d_{u}+d_{v}-2}\right)^{3}$ & augmented Zagreb index [78] & $A Z I$ \\
\hline
\end{tabular}

A graph of order $n$ is also called an $n$-vertex graph. As usual, by $P_{n}, S_{n}, C_{n}$, and $K_{n}$ we denote the $n$-vertex path, star, cycle, and complete graphs, respectively. A graph of maximum degree at most 4 is known as a chemical graph. 
Degree set of a graph $G$ is the set of all different vertex degrees of $G$. A graph whose degree set consists of only two elements is known as a bidegreed graph.

A vertex of degree 1 in a graph is known as a pendent vertex. A pendent edge of a graph is an edge incident with a pendent vertex. A vertex of degree greater than 2 in a graph is called a branching vertex. The minimum non-pendent vertex degree of a graph $G$ of is equal to 2 .

A graph $G$ containing no cycles is an acyclic graph. The minimum number of edges of a graph $G$ whose removal makes $G$ acyclic is its cyclomatic number. By a $\nu$-cyclic graph, we mean a graph having cyclomatic number $\nu$. We refer 1-cyclic, 2-cyclic, 3-cyclic, 4-cyclic graphs as unicyclic, bicyclic, tricyclic, tetracyclic graphs, respectively.

The complement of a graph $G$, denoted by $\bar{G}$, is the graph with the vertex set $V(\bar{G})=V(G)$ and $u v \in E(\bar{G})$ if and only if $u v \notin E(G)$. The union $H \cup K$ of two graphs $H$ and $K$ is the graph with the vertex set $V(H) \cup V(K)$ and the edge set $E(H) \cup E(K)$. Two graphs $H$ and $K$ satisfying the constraint $V(H) \cap V(K)=\emptyset$ are called disjoint. In this paper, whenever we use the notion of union, it would be understood that the graphs on which this operation is applied, are pairwise disjoint. The join of two graphs $H$ and $K$, denoted by $H+K$, is the graph obtained from $G \cup K$ by joining each vertex of $G$ with every vertex of $K$ through an edge.

The eccentricity of a vertex $v$ in a graph is the distance from $v$ to a vertex furthest from $v$. The radius and diameter of a graph $G$ are the minimum number and maximum number, respectively, among all the vertex eccentricities of $G$.

A graph $G$ whose vertex set can be partitioned into $r \geq 2$ subsets $A_{1}, A_{2}, \ldots, A_{r}$ in such a way that all the vertices of $A_{i}$ are pairwise non-adjacent for $i=1,2, \ldots, r$, is called $r$-partite graph, and in this case the sets $A_{1}, A_{2}, \ldots, A_{r}$ are called the partite sets of $G$. If, in addition, every vertex of $A_{i}, i=1,2, \ldots, r$, is adjacent to all the vertices of the other partite sets, then the graph $G$ is known as the complete r-partite graph. A (complete) 2-partite graph is also known as the (complete) bipartite graph. A complete bipartite graph having $p$ elements in one partite set and $q$ elements in the other partite set, is denoted by $K_{p, q}$ or $K_{q, p}$. A bipartite bidegreed graph in which all the vertices of each partite set have the same degree is called semiregular bipartite graph.

A $k$-polygonal system is a connected geometric figure obtained by concatenating congruent regular $k$-polygons side to side in a plane in such a way that the figure divides the plane into one infinite (external) region and several finite (internal) regions, and all internal regions must be congruent regular $k$-polygons. Two polygons, in a $k$-polygonal system, that have a common side are called adjacent polygons. The characteristic graph of a $k$-polygonal system is a graph $C G$ whose vertices correspond to $k$-polygons of the system and two vertices of $C G$ are adjacent if and only if the corresponding $k$-polygons are adjacent. A $k$-polygonal system whose characteristic graph is the path graph (respectively, tree) is known as $k$-polygonal chain (respectively, catacondensed k-polygonal system).

In a $k$-polygonal chain, a $k$-polygon adjacent to exactly one (respectively, two) $k$-polygon(s) is called external (respectively, internal) $k$-polygon. Any $k$-polygonal system can be represented by a graph, in which the edges correspond to the sides of a $k$-polygon and the vertices represent the points where two sides of a $k$-polygon meet. In the remaining part of this survey paper, by a $k$-polygonal system we mean the graph corresponding to the $k$-polygonal system.

A 3-polygonal (triangular) chain in which every vertex has degree at most 4 is called a linear triangular chain. (Note that for every triangular chain $T_{n}$, there exist a 6-polygonal (hexagonal) system whose characteristic graph is isomorphic to $T_{n}$.)

A subgraph $H$ of a graph $G$ satisfying the following property is called an induced subgraph: uv $\in E(H)$ whenever $u, v \in V(H)$ and $u v \in E(G)$. An induced subgraph of a triangular chain $T_{n}$ is said to be a segment if it is a maximal linear triangular sub-chain of $T_{n}$. A segment containing external triangle(s) is called external segment. Suppose that a triangular chain $T_{n}$ has $s$ segments $S_{1}, S_{2}, S_{3}, \ldots, S_{s}$. The number of triangles in a segment $S_{i}$ (where $1 \leq i \leq s$ ) is its length. The $s$-tuple $\left(a_{1}, a_{2}, \ldots, a_{s}\right)$ is said to be the length vector of $T_{n}$ if and only if $a_{i}$ is the length of $S_{i}$ for $i=1,2, \ldots, s$. If $\left(a_{1}, a_{2}, \ldots, a_{s}\right)$ is a length vector of $T_{n}$ and $s \geq 3$, then we assume that $a_{1}, a_{s}$ are the lengths of the external segments. By a zigzag triangular chain, we mean a chain with $n \geq 4$ triangles and length vector

$$
(a, \underbrace{4,4,4, \cdots, 4}_{\left(\left\lfloor\frac{n}{2}\right\rfloor-2\right)-\text { times }}, b)
$$

where $a, b \leq 4$ and at least one of $a, b$ is 3 .

In a 4-polygonal (polyomino) chain, an internal square having a vertex of degree 2 is known as a kink. In a 5polygonal (pentagonal)/6-polygonal (hexagonal) chain, a kink is an internal pentagon/hexagon that contains an edge connecting vertices of degree 2. A linear polyomino/pentagonal/hexagonal chain is the one, without kinks. A zigzag polyomino/pentagonal/hexagonal chain is the one, consisting of only kinks and external polygons. A segment in a polyomino/pentagonal/hexagonal chain is a maximal linear sub-chain, including the kinks and/or external polygons at its 
ends. The number of polygons in a segment is called its length. A segment is said to be external (internal, respectively) if it contains (does not contain, respectively) external polygons. Two segments that have a polygon in common are called adjacent segments.

In a catacondensed hexagonal system, a hexagon adjacent to three other hexagons is referred to as a branched hexagon.

The hexagonal systems with an equal number of hexagons and an equal number of internal vertices are isomeric. Also, isomeric hexagonal systems have an equal number of vertices and equal number of edges. Paths along the perimeter of a hexagonal system having degree sequences $(2,3,2),(2,3,3,2),(2,3,3,3,2),(2,3,3,3,3,2)$ are known as fissure, bay, cove, fjord, respectively. The sum of the number of fissures, bays, coves, and fjords of a hexagonal system $S$ is its number of inlets.

Let $H_{1}$ and $H_{2}$ be two hexagonal systems and let $u, v \in V\left(H_{1}\right)$ be vertices of degree 2, separated by a degree 3 vertex. Let $x, y \in V\left(H_{2}\right)$ be two adjacent vertices of degree 2. A fluoranthene system $F$ is a chemical graph constructed from the hexagonal systems $H_{1}$ and $H_{2}$ by adding the edges $u x$ and $v y$. If, in addition, $F$ satisfying the following three conditions then $F$ is called a fluoranthene linear chain: (i) $H_{1}$ has only two hexagons (ii) $H_{2}$ is the hexagonal linear chain (iii) each of the vertices $x, y$ is adjacent to only vertices of degree 2 in $H_{2}$.

\section{Bounds}

Let $G$ be a graph with a given set $\mathcal{A}$ of graph invariants (for example, size, order, minimum degree, etc.) and suppose that $\Theta$ is a bound on $A B C(G)$ in terms of all the elements of $\mathcal{A}$. We say that the bound $\Theta$ is the best possible if and only if for every choice of the values of the parameters of $\mathcal{A}$ there exists at least one graph attaining this bound. In this section, we list only those bounds on the $A B C$ index that are not best possible over the given graph family, or better to say, we present bounds on the $A B C$ index for some quite general graph classes in this section. However, if one restricts his/her observation to more special classes of graphs, then sharper results may hold in such specific cases. These kinds of results will be presented in Section 4, under the topic "extremal results".

\subsection{Upper bounds on the atom-bond connectivity index}

Recall that a graph with the maximum degree at most 4 is called a chemical graph. In the seminal paper [77], an upper bound on the $A B C$ index for an arbitrary chemical tree in terms of its order was derived.

Theorem 3.1. [77] If $T$ is a chemical tree of order $n$, then

$$
A B C(T) \leq \frac{\sqrt{3}}{4}(n+3)+\frac{\sqrt{2}}{4}(n-5) .
$$

If $n \geq 9$ with $n \equiv 1(\bmod 4)$ and if $T$ is isomorphic to the tree depicted in Figure 1, then equality in (2) holds.

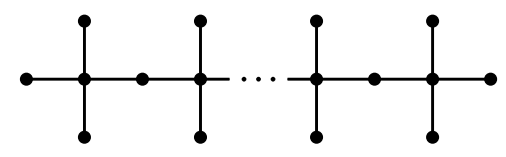

Figure 1: A tree of order $n$ for which equality in (2) holds, where $n \equiv 1(\bmod 4)$ and $n \geq 9$.

The following result is an extension of Theorem 3.1 for chemical bicyclic graphs. Indeed, both of these two results can be considered as the special cases of some generalized results, see for example Theorem 3.5 and Corollary 3.1.

Theorem 3.2. [22] If $G$ is a connected chemical bicyclic graph of order $n$, then

$$
A B C(G) \leq \frac{\sqrt{3}}{4}(n-3)+\frac{\sqrt{2}}{4}(n+5) .
$$

If $n \geq 23$ with $n \equiv 3(\bmod 4)$ and if $G$ is isomorphic to the graph depicted in Figure 2, then equality in (3) holds.

The next two results give the upper bounds on the $A B C$ index of chemical trees and connected chemical unicyclic graphs, in terms of their order and number of pendent vertices.

Theorem 3.3. [145] If $T$ is a chemical tree with $n$ vertices and $n_{1} \geq 5$ pendent vertices, then

$$
A B C(T) \leq \frac{1}{2}\left(\sqrt{2} n+(\sqrt{3}-\sqrt{2}) n_{1}-\sqrt{2}\right)
$$




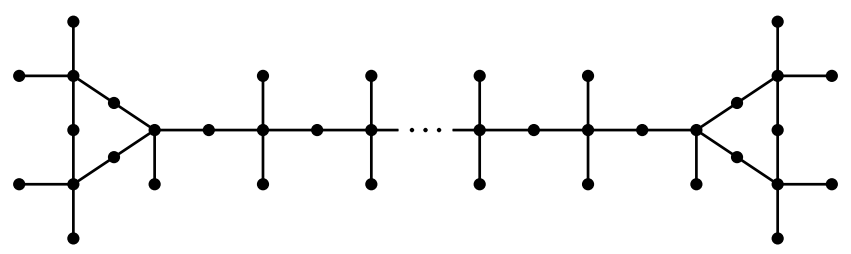

Figure 2: A graph of order $n$ for which equality in (3) holds, where $n \equiv 3(\bmod 4)$ and $n \geq 23$.

with equality if and only if $m_{1,4}=n_{1}, m_{2,2}=n-2 n_{1}-\frac{1}{3} m_{2,3}+3, m_{2,4}=n_{1}-\frac{2}{3} m_{2,3}-4, n_{2}=n-\frac{3}{2} n_{1}-\frac{1}{6} m_{2,3}+1, n_{3}=\frac{1}{3} m_{2,3}$, and $n_{4}=\frac{1}{2} n_{1}-\frac{1}{6} m_{2,3}-1$; where $m_{i, j}$ is the number of those edges of $T$ whose one end-vertex has degree $i$ and the other end-vertex has degree $j$, and $n_{i}$ is the number of vertices of $T$ having degree $i$.

Theorem 3.4. [80] If $G$ is a connected unicyclic chemical graph of order $n \geq 3$ and with $n_{1}$ pendent vertices, then

$$
A B C(G) \leq \frac{n}{\sqrt{2}}+\frac{n_{1}(\sqrt{3}-\sqrt{2})}{2}
$$

with equality if and only if every pendent edge is incident with a vertex of degree 4 and at least one end-vertex of every non-pendent edge has degree 2.

If $G^{\prime}$ is a graph formed from another graph $G$ by removing an edge $u v \in E(G)$ and adding a new vertex $w$ together with the two edges $u w$ and $w v$, then we say that $G^{\prime}$ is obtained from $G$ by subdividing the edge $u v$. For $n \geq 5$, Theorem 3.1 follows also from next theorem (for detail, see [145]).

Theorem 3.5. [145] If $T$ is tree of order $n \geq 3$ and maximum degree $\Delta$, then

$$
A B C(G) \leq \sqrt{1-\frac{1}{\Delta}}\left(n-\frac{2(n-1)}{\Delta}+1\right)+\sqrt{2}\left(\frac{n-1}{\Delta}-1\right) .
$$

with equality if and only if $G$ is the path graph for $\Delta=2$, or $G \in \mathcal{T}_{n, \Delta}$ with $n \equiv 1(\bmod \Delta)$ for $\Delta \geq 3$, where $\mathcal{T}_{n, n-1}=\left\{S_{n}\right\}$, and for $3 \leq \Delta \leq n-2, \mathcal{T}_{n, \Delta}$ is the class of trees obtained by subdividing every edge of a tree of order $\frac{n-1}{\Delta}$ with maximum degree at most $\Delta$, whose vertices are denoted by $v_{1}, v_{2}, \cdots, v_{\frac{n-1}{\Delta}}$, and then attaching some pendent vertices to $v_{i}$ until the degree of $v_{i}$ is equal to $\Delta$ for $i=1,2, \cdots, \frac{n-1}{\Delta}$.

Note that if $n \equiv 1(\bmod 4)$, then equality in (2) holds not only for the tree shown in Figure 1, but also for every tree satisfying $m_{2,4}=(n-5) / 2$ and $m_{1,4}=(n+3) / 2$, where $m_{i, j}$ is the number of those edges of the tree whose one end-vertex has degree $i$ and the other end-vertex has degree $j$. Indeed, for $n \geq 5$, the equality in (2) holds if and only if $T \in \mathcal{T}_{n, 4}$ with $n \equiv 1(\bmod 4)$ (for detail, see [145]), where the graph class $\mathcal{T}_{n, 4}$ is defined in Theorem 3.5. The next result proved independently in [80,144], is a generalization of Theorem 3.5.

Theorem 3.6. $[80,144]$ If $G$ is a connected graph of order $n$, size $m$ and maximum degree $\Delta \geq 3$, then

$$
A B C(G) \leq\left(2 n-\frac{2 m}{\Delta}-m\right) \sqrt{1-\frac{1}{\Delta}}+\sqrt{2}\left(\frac{m}{\Delta}+m-n\right)
$$

with equality if and only if $m \equiv 0(\bmod \Delta)$ and one end-vertex of every edge of $G$ has degree $\Delta$ and the other end-vertex has degree 1 or 2 .

Note that the bound on the $A B C$ index given in Theorem 3.6 is not sharp if either $m>2 n-\frac{2 m}{\Delta}$ or $m \not \equiv 0(\bmod \Delta)$. The corollary given next is a direct consequence of Theorem 3.6.

Corollary 3.1. [144] Let $G$ be a connected chemical graph of order $n \geq 5$ and size $m$.

(i) If $n-1 \leq m \leq\left\lfloor\frac{12\left(\sqrt{3}-2 \sqrt{\frac{2}{3}}\right)}{9 \sqrt{3}+\sqrt{2}-20 \sqrt{\frac{2}{3}}} n\right\rfloor$

$$
A B C(G) \leq(\sqrt{3}-\sqrt{2}) n+\left(\frac{5 \sqrt{2}-3 \sqrt{3}}{4}\right) m
$$

with equality if and only if one end-vertex of every edge of $G$ has degree 4 and the other end-vertex has degree 1 or 2.

(ii) If $\left\lfloor\frac{12\left(\sqrt{3}-2 \sqrt{\frac{2}{3}}\right)}{9 \sqrt{3}+\sqrt{2}-20 \sqrt{\frac{2}{3}}} n\right\rfloor \leq m \leq 2 n$

$$
A B C(G) \leq\left(2 \sqrt{\frac{2}{3}}-\sqrt{2}\right) n+\left(\frac{3 \sqrt{2}-5 \sqrt{\frac{2}{3}}}{3}\right) m
$$

with equality if and only if one end-vertex of every edge of $G$ has degree 3 and the other end-vertex has degree 1 or 2. 
Note that Corollary 3.1 generalizes Theorems 3.1 and 3.2.

A graph that can be drawn in such a way that no pair of edges intersect each other, except possibly at their end-vertices, is called planar graph. The next result gives simple upper bounds on the $A B C$ index for connected graphs, $c$-cyclic connected graphs, and planar connected graphs.

Theorem 3.7. [121] (i) If $G$ is a connected graph of order $n \geq 3$, then

$$
A B C(G) \leq(n-1) \sqrt{\frac{n}{2}} .
$$

(ii) If $c$ is a non-negative integer and if $G$ is a c-cyclic connected graph of order $n \geq 3$, then

$$
A B C(G) \leq \sqrt{(n-1)(n-1+c)} .
$$

(ii) If $G$ is a planar connected graph of order $n \geq 3$, then

$$
A B C(G) \leq \sqrt{3(n-2)(n-1)} .
$$

The next two theorems give upper bounds on the $A B C$ index in terms of size, maximum degree, number of pendent vertices, and number of non-pendent edges having at least one end-vertex of degree 2, or minimum non-pendent vertex degree, of the considered graph.

Theorem 3.8. [80] Let $G$ be a connected graph of size $m$, and maximum degree $\Delta$. Also, let pand $l$ be its number of pendent vertices and number of non-pendent edges having at least one end-vertex of degree 2 , respectively. Then

$$
A B C(G) \leq \frac{2(m-l-p)}{3}+p \sqrt{\frac{\Delta-1}{\Delta}}+\frac{l}{\sqrt{2}}
$$

with equality if and only if either $G \in \mathcal{G}_{1}$ or $G \in \mathcal{G}_{3}$, where $\mathcal{G}_{3}$ is the class of connected graphs of size m, maximum degree $\Delta \geq 3$ and with p pendent vertices, such that both the end-vertices of exactly $s \geq 1$ edges have degree 3 , every pendent edge is incident with a vertex of degree $\Delta$ and at least one end-vertex of each of the remaining $m-s-p$ edges has degree 2 , and $\mathcal{G}_{1}$ is defined in Theorem 3.9. (Note that if $p=0$ and $m=s$ then the class $\mathcal{G}_{3}$ consists of 3-regular graphs.)

Theorem 3.9. [38] Let $G$ be a connected graph of order $n \geq 3$, size $m$ and maximum degree $\Delta$. Also, let $p$ and $\delta_{1}$ be its number of pendent vertices and minimum non-pendent vertex degree, respectively. Then

$$
A B C(G) \leq \frac{(m-p) \sqrt{2\left(\delta_{1}-1\right)}}{\delta_{1}}+p \sqrt{1-\frac{1}{\Delta}}
$$

with equality if and only if $G$ is regular, or it has the degree set $\{1, \Delta\}$, or $G \in \mathcal{G}_{1}$, or $G \in \mathcal{G}_{2}$ where $\mathcal{G}_{1}$ is the class of those connected graphs in which every pendent edge is incident with a vertex of maximum degree and at least one end-vertex of each of the non-pendent edges has degree 2 , and $\mathcal{G}_{2}$ is the class of those connected graphs in which every edge has at least one end-vertex of degree 2.

An upper bound on the $A B C$ index similar to the one mentioned in Theorem 3.9 can be found in [123], see Proposition 2 of [123]. These two bounds are not comparable [123]. Next, we list some bounds on the $A B C$ index, derived in [122,123].

Theorem 3.10. [122,123] If $T$ is a tree order $n \geq 2$ and maximum degree $\Delta$, then

$$
A B C(G) \leq \frac{\sqrt{\Delta^{2}-1}}{\Delta}(n-1) .
$$

It is mentioned in [123] that all upper bounds on the $A B C$ index derived in [122], except those for trees, are not justified.

Theorem 3.11. [123] If $G$ is a connected graph order $n \geq 2$, size $m$ and maximum degree $\Delta$, then

$$
A B C(G) \leq m \sqrt{\frac{n}{m}-\frac{2}{\Delta^{2}}}
$$

with equality if and only if $G$ is a regular graph.

The bound on the $A B C$ index mentioned in Theorem 3.11 is stronger than the one given in (5) (see Theorem 3.14), and also the aforementioned bound of Theorem 3.11 is better than the one given in (4) (see Theorem 3.14) for $\delta<\Delta$ and these two bounds are same for the case $\delta=\Delta$ (for details, see Remark 1 in [123]). The following result gives a simple upper bound on the $A B C$ index in terms of the order and radius of the considered graph. 
Theorem 3.12. [66] If $G$ is a connected non-trivial graph of order $n$ and with the radius $r$, then

$$
A B C(G) \leq n \sqrt{\frac{n-2}{2}}+r-1 .
$$

with equality if and only if $G$ is the complete graph of order $n$.

The maximum number of pairwise adjacent vertices of a graph $G$ is called clique number of $G$. Now, we mention some upper bounds on the $A B C$ index, derived in [100].

Theorem 3.13. [100] If $G$ is a connected graph of order $n$, size $m$, minimum degree $\delta \geq 2$, maximum degree $\Delta$ and with the clique number $\omega$, then

$$
A B C(G) \leq\left(\frac{n^{2}(\omega-1)}{2 \omega}-\Delta\right) \frac{\sqrt{2(\delta-1)}}{\delta}+\sqrt{\frac{\Delta(\Delta+\delta-2)}{\delta}}
$$

with equality if and only if $G$ is a complete w-partite graph having all partite sets with the same cardinality.

Corollary 3.2. [100] If $G$ is a connected graph of order $n$, minimum degree $\delta \geq 2$ and with the clique number $\omega$, then

$$
A B C(G) \leq \frac{n^{2}(\omega-1)}{2 \omega} \cdot \frac{\sqrt{2(\delta-1)}}{\delta}
$$

with equality if and only if $G$ is a complete w-partite graph having all partite sets with the same cardinality.

The spectral radius of a graph $G$ is the largest absolute value of the eigenvalues of the adjacency matrix of $G$.

Corollary 3.3. [100] Let $G$ be a connected graph of order $n \geq 3$ and minimum degree $\delta \geq 2$, and if $\lambda_{1}$ is the spectral radius of $G$, then

$$
A B C(G) \leq \frac{n^{2}}{2}\left(1-\frac{1}{1+\lambda_{1}}\right) \frac{\sqrt{2(\delta-1)}}{\delta}
$$

with equality if and only if $G$ is the complete graph.

Several upper bounds on the $A B C$ index in terms of Randić index $R$, first Zagreb index $M_{1}$, second Zagreb index $M_{2}$, and modified second Zagreb index $M_{2}^{*}$ were derived in [95], however some of these bounds are erroneous and the corrected versions of most of these erroneous bounds were reported in [39].

Theorem 3.14. [39,95] (i) If $G$ is a connected graph of size $m \geq 1$, minimum degree $\delta$ and maximum degree $\Delta$, then

$$
A B C(G) \leq \frac{m \sqrt{2(\Delta-1)}}{\delta}
$$

and

$$
A B C(G)<\frac{m(\delta+\Delta)}{\sqrt{\delta \Delta}} .
$$

(ii) If $G$ is a connected graph of maximum degree $\Delta \geq 1$ and if $R$ is the Randic index of $G$, then

$$
A B C(G) \leq \sqrt{2(\Delta-1)} R .
$$

The equality in each of the two inequalities (4) and (6) holds if and only if $G$ is a regular graph.

A spectral upper bound on the $A B C$ index was reported in [72]. To state this bound, we firstly define the $A B C$ matrix. The $A B C$ matrix of a graph $G$ with the vertex set $\left\{v_{1}, v_{2}, \cdots, v_{n}\right\}$ is the square matrix of order $n$ whose $(i, j)$-entry is equal to $\left(d_{v_{i}}+d_{v_{j}}-2\right) /\left(d_{v_{i}} d_{v_{j}}\right)$ if $v_{i} v_{j} \in E(G)$, and 0 otherwise. Let $A B C(i)$ be the sum of the $i$-th row or column of the $A B C$ matrix of a graph $G$.

Theorem 3.15. [72] Let $G$ be an n-vertex graph. If $\vartheta_{1}$ is the largest eigenvalue of the $A B C$ matrix of $G$, then $A B C(G) \leq \vartheta_{1} n / 2$ with equality if and only if there is a constant $k$ such that $A B C(i)=k$ for every $i \in\{1,2, \cdots, n\}$.

Upper bounds on the $A B C$ index of graphs under several graph operations can be found in [2,27,75,100,124]. Recall that $G A(G)$ denotes the geometric-arithmetic index of a graph $G$. With the next result appeared in [45], study one the inequality $A B C(G)<G A(G)$ was initiated.

Theorem 3.16. [45] Let $G$ be a connected non-trivial graph of maximum degree $\Delta$ and minimum degree $\delta$ such that $\Delta-\delta \leq 3$. Also, if $G$ is neither isomorphic to the 5-vertex star graph nor isomorphic to the 8-vertex tree having vertices of degrees 1 and 4 only, then

$$
A B C(G)<G A(G)
$$


Some further classes of graphs for which (7) holds, can be found in the papers [131,152]. Similar results can be found in [43,79] concerning the $A B C$ index and the Graovac-Ghorbani index $A B C_{G G}$, and also see [42] for analogous results concerning the $A B C$ index and the average eccentricity - the Graovac-Ghorbani index for a graph $G$ is defined as

$$
A B C_{G G}(G)=\sum_{u v \in E(G)} \sqrt{\frac{n_{u}+n_{v}-2}{n_{u} n_{v}}}
$$

where $n_{u}$ is the number of those vertices of $G$ whose distance from the vertex $u$ is smaller than the distance from the vertex $v$, and similarly $n_{v}$ is defined, and the average eccentricity of a connected graph $G$ is the mean value of eccentricities of all vertices of $G$. In [76], it was proved that the inequality $A B C(G)<2 S D D(G) / 3$ holds for every connected graph $G$ of order 3 or more, where $S D D(G)$ is the symmetric division $\operatorname{deg}$ index of $G$ (see Table 1).

A sharp upper bound on the $A B C$ index for bicyclic graphs was derived in [23]. In the remaining part of this subsection, we gather the upper bounds on the $A B C$ index involving at least one of the topological indices mentioned in Table 1 .

Theorem 3.17. $[40,41]$ Let $G$ be a connected graph of order $n \geq 3$, size $m$ and maximum degree $\Delta$. Also, let $p, \delta_{1}, M_{1}$, and $M_{2}^{*}$ be its number of pendent vertices, minimum non-pendent vertex degree, fist Zagreb index, and modified second Zagreb index, respectively. Then

$$
A B C(G) \leq \sqrt{\left(M_{1}-2 m-p\left(\delta_{1}-1\right)\right)\left(M_{2}^{*}-\frac{p}{\Delta}\right)}+p \sqrt{1-\frac{1}{\Delta}}
$$

with equality if and only if $G$ is regular, or $G$ has the degree set $\{1, \Delta\}$, or $G$ is a semiregular bipartite graph.

Theorem 3.18. [155] If $G$ is a graph of order $n$, size $m$ and with the modified second Zagreb index $M_{2}^{*}$, then

$$
A B C(G) \leq \sqrt{m\left(n-2 M_{2}^{*}\right)}
$$

with equality if and only if $G$ satisfies one of the following conditions: (i) $m=0$, (ii) $G$ has no isolated vertices, and at least one-end vertex of every edge has degree 2 , (iii) $G$ has no isolated vertices and no vertex of degree 2, and every component of $G$ is either a regular graph of degree $r$ for all such components (if exist) or a semiregular bipartite graph with the degree set $\{s, t\}$ provided that st $/(s+t-2)$ is constant in all such components (if exist), and st $/(s+t-2)=(2 r-2) / r^{2}$ if there exist both types of the components.

Theorem 3.19. [13] If $G$ is a connected graph of order $n$, size $m$ and with the modified second Zagreb index $M_{2}^{*}$, then

$$
A B C(G) \leq \sqrt{(n-1)\left(m-M_{2}^{*}\right)}
$$

with equality if and only if $G$ is either a complete graph or a star graph.

Note that the bounds on the $A B C$ index, given in Theorems 3.18 and 3.19, involve the same parameters. However, these bounds are not comparable, in general. In addition to the bound mentioned in Theorem 3.19, several other upper bounds on the $A B C$ index were reported in [13].

Theorem 3.20. [155] Let $G$ be a graph of size $m$. Also, let $M_{1}$ and $M_{2}^{*}$ be its first Zagreb index and modified second Zagreb index, respectively. Then

$$
A B C(G) \leq \sqrt{\left(M_{1}-2 m\right) M_{2}^{*}}
$$

with equality if and only if either $m=0$, or every component of $G$ is either a regular graph of degree $r$ for all such components (if exist) or a semiregular bipartite graph with the degree set $\{s, t\}$ provided that st $/(s+t-2)$ is constant in all such components (if exist), and $s t /(s+t-2)=r^{2}(2 r-2)$ if there exist both types of the components.

The bound on the $A B C$ index given in Theorem 3.20 was derived also in [95] independently for connected graphs however, the graphs attaining this bound were not correctly determined there, and later on all these graphs were found in [39], which are the same as mentioned in Theorem 3.20.

A graph containing no cycle of length 3 is called a triangle-free graph.

Theorem 3.21. [155] If $G$ is a triangle-free graph of order $n \geq 3$, then

$$
\begin{aligned}
& A B C(G) \leq \sqrt{n-2} R, \\
& A B C(G) \leq \frac{n \sqrt{n-2}}{2},
\end{aligned}
$$

where $R$ in (8) is the Randic index of $G$. The equality in (8) holds if and only if G a complete bipartite graph, while the equality in (9) holds if and only if $G \cong K_{\frac{n}{2}, \frac{n}{2}}$. 
Theorem 3.22. [97] Let $G$ be a graph of order $n$ and size $m$. Also, let $M_{2}$ be the second Zagreb index of $G$. Then

$$
A B C(G) \leq \sqrt{m\left(n-\frac{2 m^{2}}{M_{2}}\right)}
$$

with equality if and only if $G$ is either a regular graph or a semiregular bipartite graph.

Theorem 3.23. [41] Let $G$ be a graph of order $n \geq 4$, such that its complement $\bar{G}$ is connected. Let $\Delta, \delta_{1}, p, \bar{\Delta}, \overline{\delta_{1}}$, and $\bar{p}$ denote the maximum degree, minimum non-pendent vertex degree, the number of pendent vertices in $G$ and $\bar{G}$, respectively. If $k=\min \left\{\delta_{1}, \overline{\delta_{1}}\right\}$, then

$$
A B C(G) \leq(p+\bar{p}) \sqrt{\frac{n-3}{n-2}}\left[1-\sqrt{\frac{2}{n-2}}\right]+\left(\begin{array}{l}
n \\
2
\end{array}\right) \sqrt{\frac{2(k-1)}{k^{2}}}-A B C(\bar{G}),
$$

with equality if and only if $G$ is the path graph of order 4 or $G$ is an r-regular graph with $r=\frac{n-1}{2}$.

Theorem 3.24. [153] Let $G$ be a connected graph of order $n \geq 3$. If $R, X$ and $H$ are the Randic index, sum-connectivity index and harmonic index of $G$, respectively, then

$$
\begin{gathered}
A B C(G) \leq \sqrt{2(n-1)} R, \\
A B C(G) \leq \begin{cases}\sqrt{2} X & \text { if } n=3, \\
\sqrt{\frac{8}{3}} X & \text { if } n=4, \\
\sqrt{\frac{n(n-2)}{n-1}} X & \text { if } n \geq 5,\end{cases} \\
A B C(G) \leq \begin{cases}\sqrt{2(n-2)} H & \text { if } 3 \leq n \leq 6, \\
\frac{n}{2} \sqrt{\frac{n-2}{n-1}} H & \text { if } n \geq 7,\end{cases}
\end{gathered}
$$

The equality sign in (10) holds if and only if $G$ is the complete graph. The equality sign in (11) holds if and only if $G$ is the

$$
\begin{cases}\text { complete graph } & \text { if } n=3, \\ \text { complete graph or star graph } & \text { if } n=4, \\ \text { star graph } & \text { if } n \geq 5 .\end{cases}
$$

The equality sign in (12) holds if and only if $G$ is the

$$
\begin{cases}\text { complete graph } & \text { if } 3 \leq n \leq 6, \\ \text { star graph } & \text { if } n \geq 7 .\end{cases}
$$

Some upper bounds on the $A B C$ index in terms of the harmonic index are derived in [91].

An upper bound on the $A B C$ index for chemical trees, in terms of their order and Randić index, was derived in [6] (see also [156] for related results).

Theorem 3.25. [152] (i) For the positive integers $s$, $t$ and $n$ satisfying $1 \leq s \leq t \leq n-1, t \geq 2$ and $n \geq 3$, let $G$ be an $n$-vertex connected graph of minimum degree at least $s$ and maximum degree at most $t$. If $G A$ is the geometric-arithmetic index of $G$, then

$$
A B C(G) \leq \begin{cases}\frac{\sqrt{2(s-1)}}{s} G A & \text { if } t<\sqrt{5 s^{2}-14 s+9}+2 s-3, \\ \frac{(t+s) \sqrt{t+s-2}}{2 t s} G A & \text { if } t \geq \sqrt{5 s^{2}-14 s+9}+2 s-3,\end{cases}
$$

with equality if and only if

$$
\begin{cases}G \text { is an s-regular graph } & \text { when } t<\sqrt{5 s^{2}-14 s+9}+2 s-3, \\ \text { one of the end-vertices of every edge of } G \text { has degree } s \text { and the other has degree } t & \text { when } t \geq \sqrt{5 s^{2}-14 s+9}+2 s-3 .\end{cases}
$$

(ii) If $G$ is a connected graph of order $n \geq 3$ and if $G A$ is the geometric-arithmetic index of $G$, then

$$
A B C(G) \leq \frac{n \sqrt{n-2}}{2(n-1)} G A
$$

with equality if and only if $G$ is the star graph $S_{n}$. 
The bound given in Theorem 3.25(ii) can be improved if the minimum degree of the considered graph is at least 2:

Theorem 3.26. [4] If $G$ is a connected graph of order $n \geq 3$, minimum degree at least 2 and if $G A$ is the geometric-arithmetic index of $G$, then

$$
A B C(G) \leq \frac{n+1}{4 \sqrt{n-1}} G A
$$

with equality if and only if $G$ is the cycle graph of order 3.

Theorem 3.27. [100] Let $G$ be a graph of size $m$ and minimum degree $\delta \geq 1$. If $R$ and $M_{1}$ are the Randic index and first Zagreb index, respectively, of $G$, then

$$
A B C(G) \leq \frac{M_{1}-3 m}{\delta(\sqrt{2(\delta-1)}+1)}+R
$$

with equality if and only if there exist a constant $r$ such that every component of $G$ is r-regular.

\subsection{Lower bounds on the atom-bond connectivity index}

Recall the definition of the best possible bound given at the start of Section 3. In this section, we try to gather only those lower bounds on the $A B C$ index that are not best possible over the considered graph family. Let us start from a simple, but important bound derived in [77].

Theorem 3.28. [77] If $T$ is a chemical tree of order $n$ then

$$
A B C(T) \geq \begin{cases}0 & \text { if } n \leq 2, \\ \frac{n-1}{\sqrt{2}} & \text { if } 3 \leq n \leq 9, \\ \frac{8}{\sqrt{2}}+\frac{2}{\sqrt{3}} & \text { if } n=10, \\ \frac{4 \sqrt{15}+3 \sqrt{6}+48 \sqrt{2}}{132} n+\frac{4 \sqrt{15}-63 \sqrt{6}+48 \sqrt{2}}{132} & \text { if } n \geq 11 .\end{cases}
$$

If $n$ is at least 21 and satisfies $n \equiv 10(\bmod 11)$, and if $T$ is isomorphic to the tree depicted in Figure 3 , then equality in (13) holds.

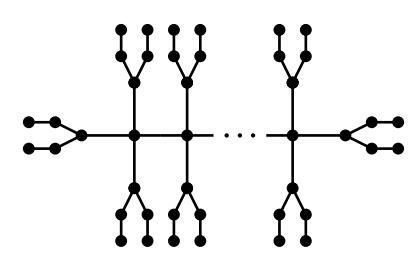

Figure 3: A tree of order $n \geq 21$ satisfying $n \equiv 10(\bmod 11)$, that attains the equality in (13).

The next two results may be considered as an extension of Theorem 3.28 for the connected chemical unicyclic and bicyclic graphs.

Theorem 3.29. [25] If $G$ is a connected chemical unicyclic graph of order $n$, then

$$
A B C(G) \geq \frac{4 \sqrt{15}+3 \sqrt{6}+48 \sqrt{2}}{132} n .
$$

If $n$ is at least 33 and satisfies $n \equiv 0(\bmod 11)$, and if $G$ is isomorphic to the graph given in Figure 4 , then equality in (14) holds.

Theorem 3.30. [22] If $G$ is a connected chemical bicyclic graph of order $n$, then

$$
A B C(G) \geq \frac{4 \sqrt{15}+3 \sqrt{6}+48 \sqrt{2}}{132} n-\frac{4 \sqrt{15}-63 \sqrt{6}+48 \sqrt{2}}{132} .
$$

If $n$ is greater than 55 and satisfies $n \equiv 1(\bmod 11)$, and if $G$ is isomorphic to the graph shown in Figure 5 , then equality in (15) holds. 


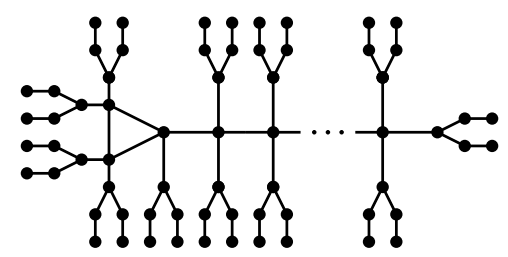

Figure 4: A graph of order $n \geq 33$ satisfying $n \equiv 0(\bmod 11)$, that attains the equality in (14).

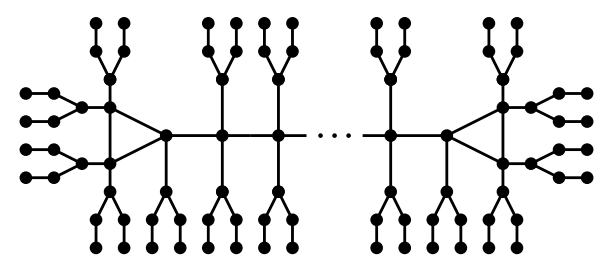

Figure 5: A graph of order $n \geq 56$ satisfying $n \equiv 1(\bmod 11)$, that attains the equality in (15).

Theorem 3.31. [80] Let $G$ be a connected graph of size $m$ and maximum degree $\Delta$. Also, let $p$ and $l$ be its number of pendent vertices and number of non-pendent edges having at least one end-vertex of degree 2, respectively. Then

$$
A B C(G) \geq \frac{p+l}{\sqrt{2}}+(m-l-p) \frac{\sqrt{2(\Delta-1)}}{\Delta}
$$

with equality if and only if $G$ is a regular graph, or a path graph, or $G \in \mathcal{G}_{\Delta, m}^{*}$, or $G \in \mathcal{G}_{2}$, where $\mathcal{G}_{\Delta, m}^{*}$ is the class of connected graphs of size m, minimum degree 2 and maximum degree $\Delta \geq 3$, such that both the end-vertices of exactly $r \geq 1$ edges have degree $\Delta$ and at least one end-vertex of each of the remaining $m-r$ edges has degree 2 , and $\mathcal{G}_{2}$ is defined in Theorem 3.9.

Corollary 3.4. [80] If $G$ is a connected graph of size $\geq 1$ and maximum degree $\Delta$, then

$$
A B C(G) \geq \frac{m \sqrt{2(\Delta-1)}}{\Delta}
$$

with equality if and only if $G$ is either a regular graph, or a path graph.

The lower bound on the $A B C$ index given in Corollary 3.4 is better than the one given in Theorem 3.33(ii), for detail see [80].

Theorem 3.32. [38] Let $G$ be a connected graph of order $n \geq 3$, size $m$ and maximum degree $\Delta$. Also, let $p$, $M_{2}^{*}$ and $\delta_{1}$ be its number of pendent vertices, modified second Zagreb index and minimum non-pendent vertex degree, respectively. Then

$$
A B C(G) \geq \frac{\sqrt{4\left(n-2 M_{2}^{*}-p\left(1-\frac{1}{\Delta}\right)\right)(m-p) \sqrt{(\Delta-1)\left(\delta_{1}-1\right)}}}{\sqrt{\delta_{1} \Delta}\left(\frac{1}{\delta_{1}} \sqrt{\delta_{1}-1}+\frac{1}{\Delta} \sqrt{\Delta-1}\right)}+p \sqrt{1-\frac{1}{\delta_{1}}}
$$

with equality if and only if $G$ is regular, or it has the degree set $\{1, \Delta\}$, or $G \in \mathcal{G}_{\Delta, m}$, where $\mathcal{G}_{\Delta, m}$ is the class of connected graphs of size $m$, minimum degree 2 and maximum degree $\Delta \geq 3$, such that both the end-vertices of exactly $m \Delta /(\Delta+2 \sqrt{\Delta-1})$ edges have degree $\Delta$ and at least one end-vertex of each of the remaining edges has degree 2.

Theorem 3.33. [38] (i) Let $G$ be a connected graph of order $n \geq 3$, size $m$ and maximum degree $\Delta$. If $M_{2}^{*}$ is the modified second Zagreb index of $G$, then

$$
A B C(G) \geq \frac{\sqrt{4 m \sqrt{2 \Delta}\left(n-2 M_{2}^{*}\right)}}{\sqrt{\Delta}+\sqrt{2}}
$$

with equality if and only if either $G$ is a path graph, or $G$ has the degree set $\{1, \Delta\}$ provided that its number of pendent vertices is $\sqrt{2} m /(\sqrt{\Delta}+\sqrt{2})$.

(ii) If $G$ is a connected graph of size $m \geq 2$ and maximum degree $\Delta$, then

$$
A B C(G) \geq \frac{2^{7 / 4} m \sqrt{\Delta-1}}{\Delta^{3 / 4}(\sqrt{\Delta}+\sqrt{2})}
$$

with equality if and only if $G$ is a path graph of order $m+1$.

For $r \geq 2$, a path $P: v_{1} v_{2} \cdots v_{r}$ in a graph is called pendent path if one of the vertices $v_{1}$ and $v_{r}$ has degree 1 and the other has degree greater than 2 , and every other vertex (if exist) of $P$ has degree 2 . 
Theorem 3.34. [18] For $n \geq 3$, if $G$ is an n-vertex connected graph having no pendent path of length at least 2 , then

$$
A B C(G) \geq \sqrt{\frac{n\left(n-2 M_{2}^{*}\right)}{2}},
$$

where $M_{2}^{*}$ is the modified second Zagreb index of G.

Theorem 3.35. [41] Let $G$ be a connected graph of order $n \geq 4$, minimum degree $\delta$ and maximum degree $\Delta$. If $k=$ $\max \{\Delta, n-\delta-1\}$ and the complement graph $\bar{G}$ of $G$ is connected, then

$$
A B C(G) \geq \frac{2^{3 / 4} n(n-1) \sqrt{k-1}}{k^{3 / 4}(\sqrt{k}+\sqrt{2})}+A B C(\bar{G})
$$

with equality if and only if $G$ is the path graph of order 4 .

The minimum number of colors required to color the vertices of a graph $G$ so that no pair of adjacent vertices have the same color is called chromatic number of $G$.

Theorem 3.36. [153] (i) Let $G$ be a connected graph of order $n \geq 3$ and minimum degree at least $k$. If $R, X$ and $H$ are the Randić index, sum-connectivity index and harmonic index of $G$, respectively, then

$$
\begin{gathered}
A B C(G) \geq \begin{cases}R & \text { if } k=1, \\
\sqrt{2(k-1)} R & \text { if } k \geq 2,\end{cases} \\
A B C(G) \geq \begin{cases}\sqrt{\frac{3}{2}} X & \text { if } k=1, \\
\sqrt{\frac{4(k-1)}{k}} X & \text { if } k \geq 2,\end{cases} \\
A B C(G) \geq \begin{cases}\frac{3}{2 \sqrt{2} H} & \text { if } k=1, \\
\sqrt{2(k-1)} X & \text { if } k \geq 2,\end{cases}
\end{gathered}
$$

The equality sign in either three inequalities (16), (17), (18) holds if and only if $G$ is the

$$
\begin{cases}\text { path graph of order } 3 & \text { when } k=1, \\ k \text {-regular graph } & \text { when } k \geq 2 .\end{cases}
$$

(ii) Let $G$ be a connected graph of order $n$ and minimum degree at least $k \geq 2$. If $\chi$ is the chromatic number of $G$, then

$$
A B C(G) \geq \sqrt{\frac{k-1}{2}} \chi
$$

with equality if and only if $G$ is the complete graph.

Some lower bounds on the $A B C$ index in terms of the harmonic index are derived in [91,124]. A lower bound on the $A B C$ index for a special type of chemical trees, in terms of their order and Randić index, was derived in [6].

Theorem 3.37. [152] (i) For the positive integers $t$ and $n$ satisfying $2 \leq t \leq n-1$, let $G$ be an n-vertex connected graph of maximum degree at most $t$. If $G A$ is the geometric-arithmetic index of $G$, then

$$
A B C(G) \geq \frac{\sqrt{2(t-1)}}{t} G A
$$

with equality if and only if $G$ is a t-regular graph.

(ii) If $G$ is a connected graph of order $n \geq 3$ and if $G A$ is the geometric-arithmetic index of $G$, then

$$
A B C(G) \geq \frac{\sqrt{2(n-2)}}{n-1} G A
$$

with equality if and only if $G$ is the complete graph $K_{n}$.

The bound given in Theorem 3.37(ii) was independently derived also in [4] under the constraint that the minimum degree of the considered graph is at least 2. 
Theorem 3.38. [94] Let $G$ be a connected non-trivial graph. If $H$ and $R^{\prime}$ are the harmonic index and modified Randić index, respectively, of $G$, then

$$
A B C(G) \geq \sqrt{2\left(H-R^{\prime}\right)} .
$$

with equality if and only if $G$ is the complete graph of order 2.

Theorem 3.39. [66] If $G$ is a connected non-trivial graph of order $n$ and with the radius $r$, then

$$
A B C(G) \geq \frac{n-1}{\sqrt{2}}-\left\lfloor\frac{n}{2}\right\rfloor+r .
$$

with equality if and only if $G$ is the path graph of order $n$.

Sharp lower bounds on the $A B C$ index of trees and unicyclic graphs, in terms of order and diameter of the considered graphs, were reported in [141].

Several lower bounds on the $A B C$ index in terms of Randić index, first Zagreb index, second Zagreb index, and modified second Zagreb index were derived in [95], however some of these bounds are erroneous and their corrected versions were reported in [39].

Theorem 3.40. [39,95] (i) If $G$ is a connected graph of size $m \geq 1$, minimum degree $\delta$ and maximum degree $\Delta$, then

$$
A B C(G) \geq \frac{m \sqrt{2(\delta-1)}}{\Delta} .
$$

(ii) If $G$ is a connected graph of minimum degree $\delta \geq 1$ and if $R$ is the Randic index $G$, then

$$
A B C(G) \geq \sqrt{2(\delta-1)} R .
$$

The equality in each of the two inequalities (19) and (20) holds if and only if $G$ is a regular graph.

We note that (20) is a special case of (16).

Theorem 3.41. [39] Let $G$ be a connected graph of size $m \geq 2$, minimum degree $\delta$ and maximum degree $\Delta$. If $M_{1}$ and $M_{2}^{*}$ are the first Zagreb index and modified second Zagreb index, respectively, of $G$, then

$$
A B C(G) \geq \frac{2 \delta \Delta M_{2}^{*} \sqrt{(\delta-1)(\Delta-1)}+M_{1}-2 m}{\delta \sqrt{2(\delta-1)}+\Delta \sqrt{2(\Delta-1)}}
$$

and

$$
A B C(G) \geq \frac{\sqrt{8 \delta \Delta M_{2}^{*}\left(M_{1}-2 m\right) \sqrt{(\delta-1)(\Delta-1)}}}{\delta \sqrt{2(\delta-1)}+\Delta \sqrt{2(\Delta-1)}},
$$

where the equality in (21) holds if and only if $G$ is a regular graph.

The bound on the $A B C$ index given in (21) also appeared in [100]. The next two results follow directly from (21).

Corollary 3.5. [39] Let $G$ be a connected graph of order $n \geq 3$, size $m$, minimum degree $\delta$ and maximum degree $\Delta$. If $M_{1}$ is the first Zagreb index of $G$, then

$$
A B C(G) \geq \frac{\delta n \sqrt{(\delta-1)(\Delta-1)}+M_{1}-2 m}{\delta \sqrt{2(\delta-1)}+\Delta \sqrt{2(\Delta-1)}}
$$

with equality if and only if $G$ is a regular graph.

Corollary 3.6. [100] If $G$ is a connected graph of order $n \geq 3$, size $m$, minimum degree $\delta$ and maximum degree $\Delta$, then

$$
A B C(G) \geq \frac{\frac{2 \delta m}{\Delta} \sqrt{(\delta-1)(\Delta-1)}+n \delta^{2}-2 m}{\delta \sqrt{2(\delta-1)}+\Delta \sqrt{2(\Delta-1)}}
$$

with equality if and only if $G$ is a regular graph.

Theorem 3.42. [100] If $G$ is a graph of minimum degree $\delta \geq 1$, then

$$
A B C(G) \geq \sqrt{2}\left(1-\frac{1}{\sqrt{\delta}}\right) R_{-1 / 4}
$$

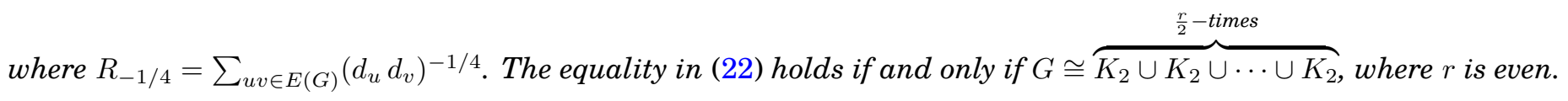


Corollary 3.7. [100] If $G$ is a graph of size $m \geq 1$, minimum degree $\delta$ and maximum degree $\Delta$, then

$$
A B C(G) \geq m \sqrt{\frac{2}{\Delta}}\left(1-\frac{1}{\sqrt{\delta}}\right)
$$

with equality if and only if $G \cong \overbrace{K_{2} \cup K_{2} \cup \cdots \cup K_{2}}^{\frac{r}{2}-\text { times }}$, where $r$ is even.

Theorem 3.43. [100] Let $G$ be a graph of size $m$ and maximum degree $\Delta \geq 1$. If $R$ and $M_{1}$ are the Randić index and first Zagreb index, respectively, of $G$, then

$$
A B C(G) \geq \frac{M_{1}-3 m}{\Delta(\sqrt{2(\Delta-1)}+1)}+R
$$

with equality if and only if there exist a constant $r$ such that every non-trivial component of $G$ is $r$-regular (a component having at least 2 vertices is called non-trivial).

In [100], the inequality $A B C(G)>r(G)$ was proved for any connected graph of order 3 or more with radius $r(G)$. We note that the inequality $A B C(G)>r(G)$ follows also from the inequality given in Theorem 3.39. Lower bounds on the $A B C$ index of graphs under several graph operations can be found in $[2,27,75,100]$.

\section{Extremal results}

This section is devoted to giving the extremal results concerning the $A B C$ index. It needs to be noted here that the best possible bounds on the $A B C$ index over particular classes of graphs follow from these extremal results.

\subsection{Extremal results regarding the maximum atom-bond connectivity index}

Studies of extremal problems concerning the maximum $A B C$ index were initiated by the paper [77], where the problem of characterizing the graph having the maximum $A B C$ index from the class of all trees of a fixed order was solved.

Theorem 4.1. [77] For every fixed integer $n \geq 4$, star graph $S_{n}$ is the unique tree with the maximum ABC index among all $n$-vertex trees, and this maximum value is $\sqrt{(n-2)(n-1)}$.

Theorem 4.1 was proved also in several other papers by using alternative ways, for example, see [38,97, 155]. From the class of all trees of a given order, the trees attaining the second, third, and fourth maximum values of the $A B C$ index were characterized in the paper [155]. The next three theorems are concerned with the maximum $A B C$ index of trees of a given order and pendent vertices or diameter.

Theorem 4.2. [155] If $n$ and $p$ are any fixed integers satisfying the inequality $3 \leq p \leq n-2$ then among all $n$-vertex trees with $p$ pendent vertices, only the tree obtained from the path graph $P_{n-p+1}$ of order $n-p+1$ by attaching $p-1$ pendent vertices to an end-vertex of $P_{n-p+1}$, has the maximum $A B C$ index, which is equal to

$$
\frac{n-p}{\sqrt{2}}+(p-1) \sqrt{\frac{p-1}{p}} .
$$

Theorem 4.2 for $p=n-2$, was also proved in $[65,149]$ independently.

Theorem 4.3. [101] Among all the trees of a fixed order $n \geq 6$ and diameter 3, only the tree obtained from the path graph $P_{3}$ of order 3 by attaching $n-3$ pendent vertices to an end-vertex of $P_{3}$, has the maximum $A B C$ index, which is equal to

$$
\sqrt{2}+(n-3) \sqrt{\frac{n-3}{n-2}} .
$$

Note that Theorem 4.3 follows directly from Theorem 4.2 because a tree $T$ of order $n \geq 6$ has $n-2$ pendent vertices if and only if $T$ has diameter 3 .

Theorem 4.4. [101] If $d$ and $n$ are any fixed integers satisfying the inequality $4 \leq d \leq n-2$ then among all $n$-vertex trees with diameter $d$, only the tree obtained from the path graph $P_{d}$ of order $d$ by attaching $n-d$ pendent vertices to an end-vertex of $P_{d}$, has the maximum $A B C$ index, which is equal to

$$
\frac{d-1}{\sqrt{2}}+(n-d) \sqrt{\frac{n-d}{n-d+1}} .
$$


Let $\mathbb{C} \mathbb{T}_{n}$ be the class of all chemical trees of a fixed order $n$ and for $i=0,1,2$, take $\mathcal{U}_{i}=\left\{T \in \mathbb{C T}_{n}: m_{1,2}=m_{2,2}=n_{3}=\right.$ $\left.0, m_{4,4}=i\right\}$ and $\mathcal{V}_{0}=\left\{T \in \mathbb{C T}_{n}: m_{1,3}=m_{1,2}=m_{2,2}=m_{4,4}=0, m_{2,3}=n_{3}=1\right\}$, where $m_{i, j}$ is the number of those edges of the tree $T$ whose one end-vertex has degree $i$ and the other end-vertex has degree $j$, and $n_{k}$ is the number of vertices of degree $k$ in $T$. Next result gives the solution to the problem of determining graph(s) having the maximum $A B C$ index among all chemical trees of a fixed order $n \geq 11$.

Theorem 4.5. [34] Among all the members of $\mathbb{C T}_{n}$, the graph(s) with the maximum $A B C$ index belong(s) to (i) $\mathcal{U}_{1}$ if $n \geq 8$ and $n \equiv 0(\bmod 4)$, and the maximum value is

$$
\frac{1}{2}\left(\sqrt{\frac{1}{2}}+\sqrt{\frac{3}{4}}\right) n+\sqrt{\frac{3}{8}}+2 \sqrt{\frac{3}{4}}-2 \sqrt{2} ;
$$

(ii) $\mathcal{U}_{0}$ if $n \geq 5$ and $n \equiv 1(\bmod 4)$, and the maximum value is

$$
\frac{1}{2}\left(\sqrt{\frac{1}{2}}+\sqrt{\frac{3}{4}}\right) n+\frac{3 \sqrt{3}}{4}-\frac{5}{2 \sqrt{2}} ;
$$

(iii) $\mathcal{V}_{0}$ if $n \geq 14$ and $n \equiv 2(\bmod 4)$, and the maximum value is

$$
\frac{1}{2}\left(\sqrt{\frac{1}{2}}+\sqrt{\frac{3}{4}}\right) n+2 \sqrt{\frac{3}{4}}-\frac{5}{\sqrt{2}}+2 \sqrt{\frac{5}{12}} ;
$$

(iv) $\mathcal{U}_{2}$ if $n \geq 11$ and $n \equiv 3(\bmod 4)$, and the maximum value is

$$
\frac{1}{2}\left(\sqrt{\frac{1}{2}}+\sqrt{\frac{3}{4}}\right) n+2 \sqrt{\frac{3}{8}}+\frac{5 \sqrt{3}}{4}-\frac{11}{2 \sqrt{2}} .
$$

A set of pairwise non-adjacent edges of a graph $G$ is called matching of $G$. A matching $M$ of a graph $G$ is called a perfect matching if every vertex of $G$ is incident with an edge of $M$.

Theorem 4.6. [145] If $k$ is a fixed integer greater than 2 then among all $2 k$-vertex trees with a perfect matching, only the tree deduced from the $k$-vertex path graph $P_{k}$ by attaching a pendent vertex to every vertex of $P_{k}$, has the maximum $A B C$ index, which is equal to

$$
\left(\sqrt{\frac{2}{3}}+\frac{2}{3}\right) k+2\left(\sqrt{2}-\sqrt{\frac{2}{3}}-1\right) .
$$

Recall the definition of the augmented Zagreb index $(A Z I)$ given in Table 1.

Theorem 4.7. [81,143] In the class of all trees of order at least 4 and with a given degree sequence, the tree(s) having a minimum $A Z I$ has a maximum $A B C$ index.

Following Hosseini et al. [98], we define a proper Kragujevac tree as a tree possessing a central vertex of degree at least 3 , to which branches of the form $B_{1}$ and/or $B_{2}$ and/or $B_{3}$ and/or $\ldots B_{k}$ are attached, where the branches $B_{1}, B_{2}, \ldots, B_{k}$ are depicted in Figure 6.

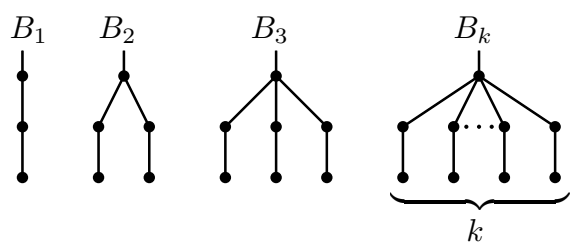

Figure 6: The branches of a proper Kragujevac tree.

Theorem 4.8. [32] Among all proper Kragujevac trees of a fixed order $n$ with the central vertex of degree $r$ (where $r \geq 3$ ) and containing no branch of type $B_{1}$, the tree $U$ has the maximum $A B C$ index, where the tree $U$ is depicted in Figure 7 .

Theorem 4.9. [33] Among all trees of a fixed order $n \geq 8$ and with exactly two branching vertices, only the tree obtained from the path graph $P_{2}$ by attaching two pendent vertices to one vertex of $P_{2}$ and $n-4$ vertices to the other vertex of $P_{2}$, attains the maximum $A B C$ index. 


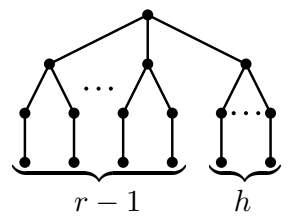

$U$

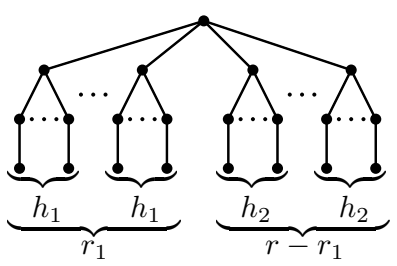

Figure 7: The trees $U$ and $V$ mentioned in Theorems 4.8 and 4.43, where $h=\frac{n-5 r+3}{2}, h_{1}=\left\lfloor\frac{n-r-1}{2 r}\right\rfloor, h_{2}=\left\lceil\frac{n-r-1}{2 r}\right\rceil$ and $r_{1}=\frac{\left(3+2 h_{1}\right) m-n+1}{2}$.

Next, we list the results concerning the maximum $A B C$ index over several classes of unicyclic graphs.

Theorem 4.10. [80,144] For $n \geq 4$, among all connected unicyclic graphs of a fixed order $n$, only the graph obtained from the $n$-vertex star $S_{n}$ by adding an edge, has the maximum ABC index, which is equal to

$$
(n-3) \sqrt{\frac{n-2}{n-1}}+\frac{3}{\sqrt{2}}
$$

From the class of all connected unicyclic graphs of a given order, the graphs attaining the second, third, and fourth maximum values of the $A B C$ index were also characterized in the paper [144] (see also [117] where the results about finding the graphs with the first two maximum values of the $A B C$ index from the aforementioned graph-class were generalized).

Theorem 4.11. [144] If $n$ and $p$ are any fixed integers satisfying the inequality $1 \leq p \leq n-3$ then among all connected unicyclic graphs of order $n$ and with p pendent vertices, only the graph obtained from the cycle graph $C_{n-p}$ of order $n-p$ by attaching $p$ pendent vertices to a vertex of $C_{n-p}$, has the maximum $A B C$ index, which is equal to

$$
\frac{n-p}{\sqrt{2}}+p \sqrt{\frac{p+1}{p+2}}
$$

If $G$ is a graph containing at least one cycle then the minimum number among the lengths of all cycles of $G$ is called girth of $G$.

Theorem 4.12. [80] Among all connected unicyclic graphs of a fixed order $n$ and with a given girth $g$, $C_{n, n-g}$ is the unique graph having the maximum $A B C$ index, which equals to

$$
(n-g) \sqrt{\frac{n-g+1}{n-g+2}}+\frac{g}{\sqrt{2}},
$$

where $C_{n, n-g}$ is the graph obtained from the cycle $C_{g}$ by attaching $n-g$ pendent vertices to one vertex of $C_{g}$.

Recall the definition of perfect matching, given just after Theorem 4.5. Graphs with the maximum $A B C$ index among all connected unicyclic graphs of a fixed order and having a perfect matching was characterized in [108].

The next two theorems are concerned with the maximum $A B C$ index of bicyclic graphs of a fixed number of pendent vertices and/or fixed order.

Theorem 4.13. [117,144] For $n \geq 5$, among all connected bicyclic graphs of fixed order $n$, only the graph obtained from the n-vertex star $S_{n}$ by adding two adjacent edges, has the maximum $A B C$ index, which is equal to

$$
(n-4) \sqrt{\frac{n-2}{n-1}}+\sqrt{\frac{n}{3(n-1)}}+2 \sqrt{2} .
$$

From the class of all connected bicyclic graphs of a fixed order $n \geq 5$, the unique graph attaining the second maximum $A B C$ index was also characterized in the paper [144].

Theorem 4.14. [144] If $p$ and $n$ are any fixed integers satisfying the inequality $0 \leq p \leq n-5$ then among all connected bicyclic graphs of order $n$ and with p pendent vertices, only the graph obtained by identifying one vertex of two cycle graphs $C_{r}$ and $C_{t}$, and by attaching $n+1-r-t$ pendent vertices to the identified vertex, has the maximum ABC index, which is equal to

$$
\frac{n+1}{\sqrt{2}}+p\left(\sqrt{\frac{p+3}{p+4}}-\frac{1}{\sqrt{2}}\right) .
$$

Now, we give the results concerning the maximum $A B C$ index of tricyclic and tetracyclic graphs with a given order. 
Theorem 4.15. [10] For $n \geq 5$, among all connected tricyclic graphs of a fixed order $n$, only the graph obtained from the $n$-vertex star $S_{n}$ by adding 3 edges between a fixed pendent vertex and 3 other pendent vertices, has the maximum ABC index, which is equal to

$$
(n-5) \sqrt{\frac{n-2}{n-1}}+\sqrt{\frac{n+1}{4(n-1)}}+3 \sqrt{2} .
$$

The graph(s) having the second and third maximum values of the $A B C$ index among all connected tricyclic graphs of a fixed order were also characterized in [10].

Theorem 4.16. [47] For $n \geq 7$, among all connected tetracyclic graphs of a fixed order $n$, only the graph obtained from the n-vertex star $S_{n}$ by adding 4 edges between a fixed pendent vertex and 4 other pendent vertices, has the maximum ABC index, which is equal to

$$
(n-6) \sqrt{\frac{n-2}{n-1}}+\sqrt{\frac{n+2}{5(n-1)}}+4 \sqrt{2} .
$$

In [47], the authors also characterized the unique graph attaining the second maximum $A B C$ index from the class of all connected tetracyclic graphs of a fixed order $n \geq 8$.

By observing Theorems 4.1, 4.10, 4.13, 4.15 and 4.16, we pose the following conjecture.

Conjecture 4.1. If $\nu$ and $n$ are any integers satisfying the inequality $5 \leq \nu \leq n-3$ then among all connected n-vertex graphs with the cyclomatic number $\nu$, only the graph deduced from the n-vertex star $S_{n}$ by adding $\nu$ edges between a fixed pendent vertex and $\nu$ other pendent vertices, has the maximum $A B C$ index, which is equal to

$$
(n-\nu-2) \sqrt{\frac{n-2}{n-1}}+\sqrt{\frac{n+\nu-2}{(\nu+1)(n-1)}}+\nu \sqrt{2} .
$$

It needs to be noted here that if $5 \leq \nu \leq n-2$ then the extremal graph mentioned in Conjecture 4.1 exists, however, the choice $\nu=n-2$ can not be included in this conjecture because of the next result (recall that $m-n=\nu-1$ where $m$ is the size of the graph).

Theorem 4.17. [132] For $n \geq 6$, among all connected graphs of a fixed order $n$, size $2 n-3$ and minimum degree at least 2 , either the extremal graph, say $H$, mentioned in Conjecture 4.1 or $K_{2, n-2}^{*}$ attains the maximum $A B C$ index, where $K_{2, n-2}^{*}$ is the graph deduced from the complete bipartite graph $K_{2, n-2}$ by adding an edge between two vertices of degree 2. More precisely, $A B C(H)>A B C\left(K_{2, n-2}^{*}\right)$ if $6 \leq n \leq 74$ and $A B C(H)<A B C\left(K_{2, n-2}^{*}\right)$ if $n \geq 75$.

The next three results are the type of Theorem 4.17, or better to say, they are related to the maximum $A B C$ index of graphs having a large size and minimum degree at least 2 .

Theorem 4.18. [132] For $n \geq 6$, among all connected graphs of a fixed order $n$, size $2 n-4$ and minimum degree at least 2 , only the complete bipartite graph $K_{2, n-2}$ attains the maximum $A B C$ index, which is equal to $\sqrt{2}(n-2)$.

Theorem 4.19. [134] For $n \geq 6$, among all connected graphs of a fixed order $n$, size $2 n-2$ and minimum degree at least 2 , either $H^{\prime}$ or $K_{2, n-2}^{* *}$ is the only graph having the maximum $A B C$ index, where $H^{\prime}$ is the graph obtained from the the extremal graph mentioned in Conjecture 4.1, by adding an edge between two vertices of degree 2 , and $K_{2, n-2}^{* *}$ is the graph deduced from $K_{2, n-2}^{*}$, defined in Theorem 4.17, by adding an edge between a vertex of degree 2 and a vertex of degree 3 . More precisely, $A B C\left(H^{\prime}\right)>A B C\left(K_{2, n-2}^{* *}\right)$ if $6 \leq n \leq 34$ and $A B C\left(H^{\prime}\right)<A B C\left(K_{2, n-2}^{* *}\right)$ if $n \geq 35$.

Theorem 4.20. [133] If $n$ and $l$ are fixed integers satisfying the inequalities $-3 \leq l \leq-1$ and $n \geq l+9$ then in the class of all bipartite connected $n$-vertex graphs of size $2 n+l$ and minimum degree at least $2, H_{n-l-9}^{3, l+6}$ is the unique graph with the maximum $A B C$ index, and this maximum value is

$$
\sqrt{2}(n-l-9)+\sqrt{\frac{(l+6)(l+7)}{3}}+2(l+6) \sqrt{\frac{n-2}{3(n-3)}},
$$

where $H_{n-l-9}^{3, l+6}$ is the graph obtained from the complete bipartite graph $K_{3, l+6}$ with bipartite sets $X=\left\{x_{1}, x_{2}, x_{3}\right\}$ and $Y=$ $\left\{y_{1}, y_{2}, \cdots, y_{l+6}\right\}$ by adding $n-l-9 \geq 0$ new vertices and joining them to $x_{1}$ and $x_{3}$.

The next theorem was independently proved in [21,40,155], however it was proved by using different ways also in several other papers, for example, see [35,49]. 
Theorem 4.21. [21,40,155] For $n \geq 3$, among all graphs of a given order $n$, only the complete graphs attains the maximum $A B C$ index, which is equal to $n \sqrt{2 n-4} / 2$.

A non-acyclic connected graph $G$ is said to be a quasi-tree graph if there exists at least one vertex $u \in V(G)$ such that $G-u$ is a tree, where $G-u$ is the graph deduced from $G$ by deleting the vertex $u$ (and all its incident edges).

Theorem 4.22. [46] For $n \geq 5$, among all connected quasi-tree graphs of a given order $n$, only the graph obtained from the star $S_{n}$ by adding $n-1$ edges between a fixed pendent vertex and $n-1$ other pendent vertices, has the maximum ABC index, which is equal to $\sqrt{2}(n-2)+\frac{\sqrt{2 n-4}}{n-1}$.

In [46], the graphs attaining the second and third maximum $A B C$ index among all connected quasi-tree graphs of a given order, were also characterized.

A cactus graph is a connected graph containing no edge lying on more than one cycle.

Theorem 4.23. $[9,65,92,104]$ If $n$ and $r$ are fixed integers satisfying the inequalities $n \geq 5$ and $0 \leq r \leq\lfloor(n-1) / 2\rfloor$ then among all $n$-vertex cactus graphs having $r$ cycles, only the graph obtained from the star $S_{n}$ by adding $r$ pairwise non-adjacent edges, has the maximum $A B C$ index, which is given as

$$
f(n, r)=(n-2 r-1) \sqrt{\frac{n-2}{n-1}}+\frac{3}{\sqrt{2}} r .
$$

Note that the function $f$ defined in Theorem 4.23, for $n \geq 5$ and $0 \leq r \leq\lfloor(n-1) / 2\rfloor$, satisfies the inequality $f(n, r) \leq$ $f(n,\lfloor(n-1) / 2\rfloor)$ with equality if and only if $r=\lfloor(n-1) / 2\rfloor$. Thus, only the graph obtained from the star $S_{n}$ by adding $\lfloor(n-1) / 2\rfloor$ pairwise non-adjacent edges, has the maximum $A B C$ index among all cactus graphs of a given order $n \geq 5$, and that maximum value is $f(n,\lfloor(n-1) / 2\rfloor)$; see [9,104] for details. From the class of all cactus graphs of a given order and with a fixed number of cycles, the unique graph having the second maximum value of $A B C$ index was also found in [9]. Moreover, the authors of [9] determined the unique graph with the second maximum value of $A B C$ index among all cactus graphs of a fixed order.

If $G^{\prime}$ is a graph formed from another graph $G$ by removing an edge $u v \in E(G)$ and adding a new vertex $w$ together with the two edges $u w$ and $w v$, then we say that $G^{\prime}$ is obtained from $G$ by subdividing the edge $u v$.

Theorem 4.24. [65,92,104] If $n$ and $p$ are fixed integers satisfying the inequalities $n \geq 5$ and $p \leq n-3$ then among all $n$-vertex cactus graphs with p pendent vertices, only the graph $G^{\prime}(n, p)$ has the maximum $A B C$ index, which is equal to

$$
\begin{cases}\frac{3}{2 \sqrt{2}}(n-p-1)+p \sqrt{\frac{n-2}{n-1}}, & \text { when } n-p \text { is odd, } \\ \frac{3}{2 \sqrt{2}}(n-p-2)+p \sqrt{\frac{n-3}{n-2}}+\frac{1}{\sqrt{2}}, & \text { when } n-p \text { is even. }\end{cases}
$$

where $G^{\prime}(n, p)$ is the graph obtained from the star

$\begin{cases}S_{n} \text { by adding }(n-p-1) / 2 \text { pairwise non-adjacent edges, } & \text { when } n-p \text { is odd, } \\ S_{n-1} \text { by adding }(n-p-2) / 2 \text { pairwise non-adjacent edges and subdividing one of these edges, } & \text { when } n-p \text { is even. }\end{cases}$

A set of pairwise non-adjacent vertices of a graph $G$ is called independent set of $G$. An independent set of a graph that covers as many vertices as possible is known as a maximum independent set. The number of elements of a maximum independent set of a graph $G$ is called independence number of $G$.

Theorem 4.25. [149] Among all connected graphs of a fixed order $n \geq 5$ and with a given independence number $\alpha$, only the graph $K_{n-\alpha}+\bar{K}_{\alpha}$ has the maximum $A B C$ index, which is equal to

$$
\frac{(n-\alpha)(n-\alpha-1) \sqrt{2(n-2)}}{2(n-1)}+\alpha \sqrt{\frac{(n-\alpha)(2 n-\alpha-3)}{n-1}} .
$$

Theorem 4.26. [149] If $n$ and $p$ are fixed integers satisfying the inequality $0 \leq p \leq n-3$ then in the class of all connected $n$-vertex graphs with $p$ pendent vertices, only the graph form by attaching $p$ pendent vertices to one vertex of $K_{n-p}$, has the maximum $A B C$ index, which is equal to

$$
\sqrt{\frac{(2 n-p-4)(n-p-1)}{n-1}}+(n-p-2) \sqrt{\frac{n-p-2}{2}}+p \sqrt{\frac{n-2}{n-1}} .
$$


The edge connectivity of a nontrivial connected graph $G$ is the minimum number of edges whose removal makes $G$ as a disconnected graph.

Theorem 4.27. [149] Among all connected graphs of a fixed order $n \geq 6$ and with a given edge-connectivity $\lambda \geq 2$, only the graph $K_{\lambda}+\left(K_{1}+K_{n-\lambda-1}\right)$ has the maximum $A B C$ index, which is equal to

$$
\sqrt{\frac{\lambda(n+\lambda-3)}{n-1}}+\frac{\lambda(\lambda-1)}{n-1} \sqrt{\frac{n-2}{2}}+\frac{(n-\lambda-2)(n-\lambda-1)}{n-2} \sqrt{\frac{n-3}{2}}+\lambda(n-\lambda-1) \sqrt{\frac{2 n-5}{(n-2)(n-1)}} .
$$

It was conjectured in [149], and later proved in the papers [37,62] independently, that the graph formed by attaching a pendent vertex to a vertex of the complete graph $K_{n-1}$, has the maximum $A B C$ index among all connected graphs of a fixed order $n \geq 4$ and with the edge-connectivity 1 . Thus, if we replace the condition " $\lambda \geq 2$ " with " $\lambda \geq 1$ " in Theorem 4.27 then the resulting statement remains true.

Recall the definition of the chromatic number a graph, given just before Theorem 3.36.

Theorem 4.28. [149] Among all connected graphs of a fixed order $n$ and with the chromatic number $\chi=2$, only the graph $T_{n, \chi}$ has the maximum $A B C$ index, which is equal to

$$
\begin{cases}\frac{n \sqrt{n-2}}{2}, & \text { when } n \text { is even, } \\ \frac{\sqrt{(n-2)\left(n^{2}-1\right)}}{2}, & \text { when } n \text { is odd. }\end{cases}
$$

where $T_{n, \chi}$ is the complete $\chi$-partite graph of order $n$ such that $\left|r_{i}-r_{j}\right| \leq 1$, where $r_{i}$, with $i=1,2, \cdots, \chi$, is the number of vertices in the $i$-th partite set of $T_{n, \chi}$.

The authors of [149] also conjectured that only the graph $T_{n, \chi}$ has the maximum $A B C$ index in the class of all connected graphs of a fixed order $n$ and with the chromatic number $\chi \geq 3$. This conjecture was proved in [62] under the constraint when $\chi$ divides $n$, and later in [19], the aforementioned conjecture was confirmed for all $\chi \geq 3$.

Recall the definition of a matching, given just after Theorem 4.5. A matching of a graph that covers as many edges as possible is known as a maximum matching. The number of elements of a maximum matching of a graph $G$ is called matching number of $G$. The vertex connectivity of a nontrivial connected graph $G$ is the minimum number of vertices whose removal makes $G$ as a disconnected or trivial graph.

In [24], the authors attacked the problems of characterizing graphs with the maximum $A B C$ index from the class of all graphs of a fixed order and with a given (i) matching number (ii) vertex connectivity. However, unfortunately, there is an error in the proof of the crucial lemma (Lemma 4) of [24] (for detail, please see Remark 2 in [20]). Thereby, these extremal problems concerning the $A B C$ index, matching number and connectivity are still open. The following result may be considered as the first step towards the solution of the aforementioned problem concerning the maximum $A B C$ index and matching number.

Theorem 4.29. [24,28] The graph attaining the maximum $A B C$ index among all connected graphs of a fixed order $n$ and with a given matching number, is of the form $K_{s}+\left(K_{n_{1}} \cup K_{n_{2}} \cup \cdots \cup K_{n_{t}}\right)$, where $s+n_{1}+n_{2}+\cdots+n_{t}=n$.

Although the proof of Theorem 1 of [24] contains an error, the following result follows from the proof of that theorem.

Theorem 4.30. [24] The graph attaining the maximum ABC index among all connected graphs of a fixed order $n$ and with a fixed vertex connectivity $\kappa$, is of the form $K_{\kappa}+\left(K_{n_{1}} \cup K_{n_{2}}\right)$, where $\kappa+n_{1}+n_{2}=n$.

We believe that statement of Theorem 1 of [24] is true, but it still needs proof.

Next, we list results concerning the maximum $A B C$ index of graphs of fixed order and with a given minimum or maximum degree.

Theorem 4.31. [44] In the class of all graphs of a fixed order $n$ and with a fixed minimum degree $\delta \geq 1$, only the graph obtained by attaching a new vertex to $\delta$ vertices of $K_{n-1}$, has the maximum $A B C$ index, which is equal to

$$
\left(\begin{array}{l}
\delta \\
2
\end{array}\right) \frac{\sqrt{2(n-2)}}{n-1}+\delta(n-\delta-1) \sqrt{\frac{2 n-5}{(n-2)(n-1)}}+\left(\begin{array}{c}
n-\delta-1 \\
2
\end{array}\right) \frac{\sqrt{2(n-3)}}{n-2}+\sqrt{\frac{\delta(n+\delta-3)}{n-1}} .
$$

Theorem 4.32. [44] For $3 \leq \Delta \leq 4$, among all graphs of a fixed order $n$ and with a fixed maximum degree $\Delta$, (i) only the regular graph(s) attain(s) the maximum $A B C$ index, which is equal to $n \sqrt{\Delta-1} / \sqrt{2}$, if $n \Delta$ is even; 
(ii) only the bidegreed graph(s) having exactly one vertex of minimum degree, which is equal to $\Delta-1$, attain(s) the maximum $A B C$ index, and this maximum value is

$$
\frac{(n \Delta-2 \Delta+1) \sqrt{2(\Delta-1)}}{2 \Delta}+\sqrt{\frac{(\Delta-1)(2 \Delta-3)}{\Delta}}
$$

if $n \Delta$ is odd.

If the condition " $3 \leq \Delta \leq 4$ " in Theorem 4.32 is replaced with " $3 \leq \Delta \leq n-1$ " then the resulting statement remains true [136].

Now, we gather results regarding the maximum $A B C$ index of $k$-polygonal systems - recall the definitions and terminology concerning the $k$-polygonal systems, given at the end of Section 2 . For the fixed integer $n \geq 4$, let $\mathfrak{T}_{n}$ be the class of all triangular (3-polygonal) chains of maximum degree at most 5 and having $n$ triangles. (It is easy to observe that $\mathfrak{T}_{n}$ form a subclass of the class of all characteristic graphs of hexagonal (6-polygonal) systems.) The next result gives the unique element of $\mathfrak{T}_{n}$ having a maximum $A B C$ index.

Theorem 4.33. [3] If $n \geq 4$ is any fixed integer then only the zigzag triangular chain has the maximum ABC index in the class $\mathfrak{T}_{n}$.

The following theorem is about the catacondensed polyomino (4-polygonal) systems - see Section 2 for the related definitions and terminology.

Theorem 4.34. [26] For every fixed integer $n \geq 3$, only the zigzag polyomino chain attains the maximum ABC index in the class of all catacondensed polyomino systems having $n$ squares.

Since the class of all polyomino chains with a fixed number of squares, say $n$, form a subclass of the class of all catacondensed polyomino systems having $n$ squares, from Theorem 4.34 it follows that for every fixed integer $n \geq 3$, only the zigzag polyomino chain attains the maximum $A B C$ index in the class of all polyomino chains having $n$ squares - this result was independently proven also in [7,36].

For any fixed integer $n \geq 3$, denote by $\Omega_{n}$ the class of all those pentagonal (5-polygonal) chains having $n$ pentagons in which every internal segment of length 3 (if it exists) contains no edge connecting the vertices of degree 3 - see Section 2 for the related definitions and terminology.

Theorem 4.35. [8] The linear pentagonal chain is the unique chain having the maximum ABC index in the class $\Omega_{n}$, for every fixed integer $n \geq 3$.

Theorem 4.36. [130] The linear pentagonal chain is the unique chain having the maximum ABC index in the class of all catacondensed pentagonal systems with $n$ pentagons, for every fixed integer $n \geq 3$.

Now, we list some results regarding hexagonal (6-polygonal) systems - these are the most studied systems among all $k$-polygonal systems in chemical graph theory.

Theorem 4.37. [31] In the class of all isomeric hexagonal systems, those having the maximum number of inlets attain the maximum value of the ABC index-see Section 2 for the related definitions.

Theorem 4.38. [21,50,102] For every fixed integer $h \geq 4$, only the linear hexagonal chain attains the maximum ABC index in the class of all catacondensed hexagonal systems having h hexagons.

Theorem 4.38 was demonstrated also in [125] independently. Further extremal results concerning the maximum $A B C$ index of certain hexagonal systems can be found from the more general results obtained in [5,11,29,30]

The next theorem is a particular case of the first part of Theorem 1 of the paper [138].

Theorem 4.39. [138] For every fixed integer $h \geq 5$, the fluoranthene linear chain attains the maximum ABC index in the class of all catacondensed fluoranthene systems having h hexagons - see Section 2 for the related definitions.

Theorem 4.39 was proved independently also in [93]. Additional extremal results concerning the maximum $A B C$ index of fluoranthene systems can be found from the general results obtained in [93]. 


\subsection{Extremal results concerning the minimum atom-bond connectivity index}

By an $n$-vertex $A B C$-minimal tree, we mean a tree having the minimum $A B C$ index in the class of all trees of a fixed order $n \geq 4$. Studies of extremal problems concerning the minimum $A B C$ index were initiated by the paper [77], where the problem of finding the $n$-vertex $A B C$-minimal tree(s) was posed. From the next result, proved independently in several papers, it is clear that the graph(s) that attain(s) the minimum $A B C$ index among all trees of a fixed order $n \geq 4$ also attain(s) the minimum $A B C$ index among all graphs of a fixed order $n \geq 4$.

Theorem 4.40. [21,40] For $n \geq 3$, among all graphs of a given order $n$, graph(s) attaining the minimum ABC index must be a tree.

The problem of finding the $n$-vertex $A B C$-minimal trees was probably one of the most studied and difficult problems in chemical graph theory in the last decade. After the seminal work about the mathematical properties of the $A B C$-minimal tree [77] many theoretical and computational results followed [1,21, 24, 38-41, 51-54, 59-61, 64, 67, 80, 81, 109, 110, 112, $113,122,155]$. The more complete list of works one can find in the following three surveys $[55,87,151]$ on this topic. After the publication of the latest survey [151] concerning the $n$-vertex $A B C$-minimal trees, several publications regarding this topic have appeared: [56,58,68,69,142] where [142] is focused on the $n$-vertex $A B C$-minimal chemical trees.

If a degree of a vertex is larger than two and no vertex of degree two is adjacent to it, then the vertex is called a big vertex. The following important property was conjectured in [1] and recently proved in [56].

Theorem 4.41. [56] The subgraph of a minimal-ABC tree induced by its big vertices is a star.

This, together with the fact that an $n$-vertex $A B C$-minimal tree with $n \geq 415$ cannot contain pendent path of length 3 [54] and together with some known properties of the degree sequences of the $A B C$-minimal trees [81,143], implies that the radius of an $n$-vertex $A B C$-minimal tree with $n \geq 415$ is at most 4 .

A vertex $v$ with degree $k+1, k \geq 2$, together with $k$ pendent paths of length 2 attached to it, comprised a so-called $B_{k}$-branch. Very recently in [57], it was claimed that another conjectured property in [1] is true:

Theorem 4.42. [57] After some enough big order n, besides the big vertices, ABC-minimal trees have only $B_{3}$-branches.

It was argued that the above theorem is true when the order of ABC-minimal tree is larger than 1014812. Moreover, in [57] it is claimed that in [1] conjectured structure of the minimal-ABC tree (see Figure 8), is true.

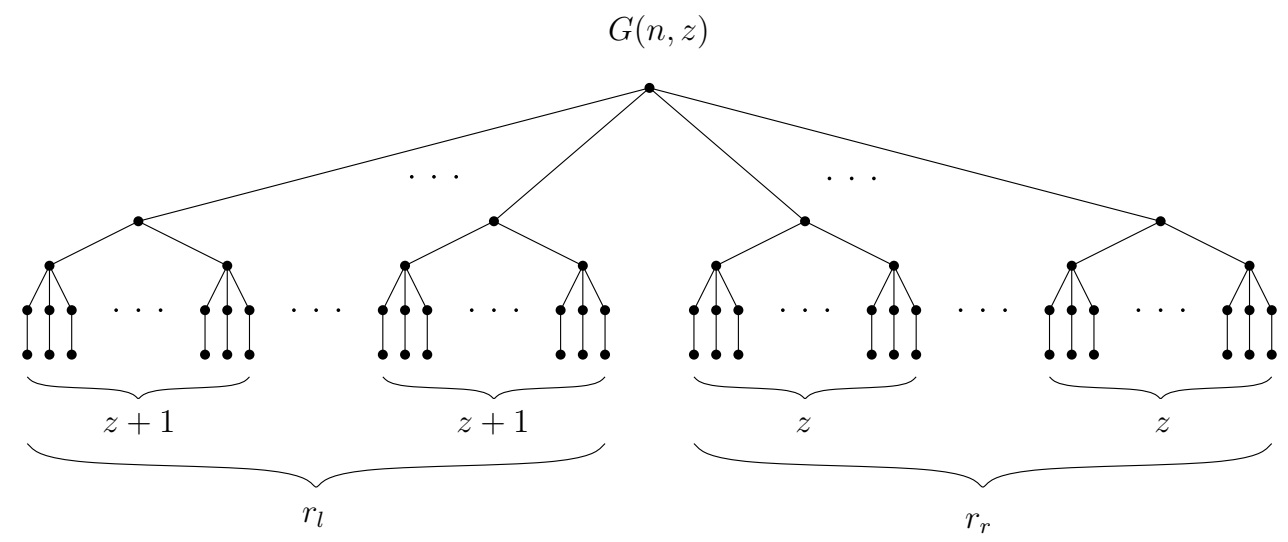

Figure 8: The figure from [1] with the conjectured structure of the minimal-ABC tree of sufficiently large order.

The best known computational performance regarding the $A B C$-minimal trees was reported in [113], where the $A B C$ minimal trees of order up to 1100 were found. Based on the obtained theoretical results, in [57] it was alleged that the $A B C$-minimal tree can be computed for any $n$ (up to some numerical limitation). With that, the problem of complete characterization of the $A B C$-minimal trees should be settled.

Recently, in a preprint [99], it has been claimed that under deleting of a certain number of vertices the structures of the $n$-vertex $A B C$-minimal trees with sufficiently large $n$ is described and that these trees have a radius at most 5 , and that all vertices except for one, have a degree at most 54.

Although the problem of finding the $n$-vertex $A B C$-minimal trees turned out to be very difficult, however, if one considers specific subclasses of the $n$-vertex trees instead of considering the class of all $n$-vertex trees then the resulting versions of the aforementioned problem concerning the minimum $A B C$ index may be solved without putting too many efforts - in the following, we list several such types of results. For the first such result, recall the definition of a proper Kragujevac tree, given just before Theorem 4.8 . 
Theorem 4.43. [32] For a fixed $n$, among all the proper Kragujevac n-vertex trees with the central vertex of degree $r$ (where $r \geq 3$ ) and containing no branches of type $B_{1}$, the tree $V$ has the minimum $A B C$ index, where the tree $V$ is depicted in Figure 7 .

A starlike tree is a tree having only one vertex of degree greater than 2.

Theorem 4.44. [12] In the class of all starlike trees of a fixed order $n \geq 7$, only those starlike tree(s) of maximum degree 3 in which the unique vertex of maximum degree has no neighbor of degree 1, attain(s) the minimum value of the ABC index.

Let $\left(d_{0}, d_{1}, \ldots, d_{n-1}\right)$ be a non-increasing degree sequence of a connected graph $G$ with the vertex set $V(G)=\left\{v_{0}, v_{1}, \ldots, v_{n-1}\right\}$ where $d_{i}=d_{v_{i}}$ for $i=0,1, \ldots, n-1$. Following Li et al. [106], we define an ordering of the vertices of $G$ induced by breadthfirst search (BFS): create a sorted list of vertices beginning with $v_{0}$; append all neighbors $u_{1}, u_{2}, \ldots, u_{d_{0}}$ of $v_{0}$ sorted by decreasing degrees; then append all neighbors of $u_{1}$ that are not already in the list, also sorted by decreasing degrees; continue recursively with $u_{2}, u_{3}, \ldots$, until all vertices of $G$ are processed. In this way, we get a rooted graph, with root $v_{0}$. The distance $d\left(v, v_{0}\right)$ is called the height $h(v)$ of a vertex $v \in V(G)$.

Let $G$ be a connected rooted graph with root $v_{0}$. A well ordering $\prec$ of the vertices is called breadth-first searching ordering [14,146] with non-increasing degrees (BFS ordering for short) if the following conditions hold for all vertices $u, v \in V(G):$

(i) $u \prec v$ implies $h(u) \leq h(v)$;

(ii) $u \prec v$ implies $d(u) \geq d(v)$;

(iii) let $u v, x y \in E(G)$ and $u y, x v \notin E(G)$ with $h(u)=h(x)=h(v)-1=h(y)-1$. If $u \prec x$, then $v \prec y$.

A graph having a BFS ordering of its vertices is known as a BFS graph.

Theorem 4.45. [81,143] Among all trees of order at least 4 and with a given degree sequence, there exists a BFS tree with the minimum $A B C$ index.

Theorem 4.45 was proved also in [150] by using an alternative technique. Lin et al. [110] generalized Theorem 4.45 for the class of connected graphs.

Theorem 4.46. [110] Among all connected graphs of order at least 4 and with a fixed degree sequence, there exists a BFS graph with the minimum $A B C$ index.

Zhang et al. [148] extended Theorem 4.46 and constructed a special BFS graph having the minimum $A B C$ index in the class all connected graphs of order at least 4 and with a fixed degree sequence. The authors of [148] also discussed the results concerning the minimum $A B C$ index of unicyclic and bicyclic graphs having a fixed degree sequence - see also Remark 4.4 of [116] as well as Corollary 5.5 in [147].

In what follows, by a $p$ - $A B C$-minimal tree we mean a tree having the minimum $A B C$ index among all trees with a fixed number $p$ of pendent vertices. The problem of finding $p$ - $A B C$-minimal trees was initiated by Goubko and Gutman in [83], where they determined all the $p-A B C$-minimal chemical trees for $p \geq 3$. This problem was attacked in several papers [82,84,109,111,118,119] and it was solved completely in [119]. Particularly, Magnant et al. [118] claimed that they had determined all the $p$ - $A B C$-minimal trees for $p \geq 19$, but this claim was refuted by Goubko et al. in [84], where they found all the $p-A B C$-minimal trees for $p \leq 53$. Later on, Gao and Shao [82] developed an algorithm for finding $p$ - $A B C$ minimal trees, and by using this algorithm, they found all the $p-A B C$-minimal trees for $2 \leq p \leq 1000$ (this range for $p$ can be extended, for example, $2 \leq p \leq 1194)$. Finally, all the $p$ - $A B C$-minimal trees for $p \geq 1195$, were determined by Mohar in [119].

Now, we turn our attention to the results concerning the minimum $A B C$ index of $k$-polygonal systems - for this we need the definitions and terminology concerning the $k$-polygonal systems, given at the end of Section 2 . Firstly, we give a result regarding the minimum $A B C$ index of polyomino (4-polygonal) chains.

Theorem 4.47. [36] For every fixed odd integer $n \geq 3$, the unique polyomino chain having segments only of length 3 provided that every internal segment (if exists) contains an edge connecting the vertices of degree 3 , has the minimum ABC index among all polyomino chains with $n$ squares.

Recall the definition of the graph class $\Omega_{n}$, given just before Theorem 4.35. For any fixed integer $n \geq 4$, denote by $\mathbb{P}_{n}$ the subclass of $\Omega_{n}$ containing all those non-linear pentagonal (5-polygonal) chains in which both the external segments have lengths less than 4 and every internal segment (if it exists) has length either 2 or 4.

Theorem 4.48. [8] For every fixed integer $n \geq 4$, the chain(s) having the minimum ABC index among all the members of $\Omega_{n}$, belong(s) to $\mathbb{P}_{n}$. 
Next, we gather some results regarding hexagonal (6-polygonal) systems.

Theorem 4.49. [31] In the class of all isomeric hexagonal systems, those having a minimum number of inlets attain the minimum value of the $A B C$ index.

Theorem 4.50. [21,50,102] For every fixed integer $h \geq 4$, the catacondensed hexagonal system with $\lfloor h / 2\rfloor-1$ branched hexagons and $\lceil h / 2-\lfloor h / 2\rfloor\rceil$ kinks is the unique system attaining the minimum $A B C$ index in the class of all catacondensed hexagonal systems having $h$ hexagons.

Theorem 4.50 was demonstrated also in [125] independently. Further extremal results concerning the minimum $A B C$ index of certain hexagonal systems can be found from the general results obtained in [5].

The next theorem is a particular case of the last two parts of Theorem 2 of the paper [138].

Theorem 4.51. [138] For every fixed integer $h \geq 5$, the system depicted in Figure 9 attains the minimum ABC index in the class of all catacondensed fluoranthene systems having h hexagons - see Section 2 for the related definitions.

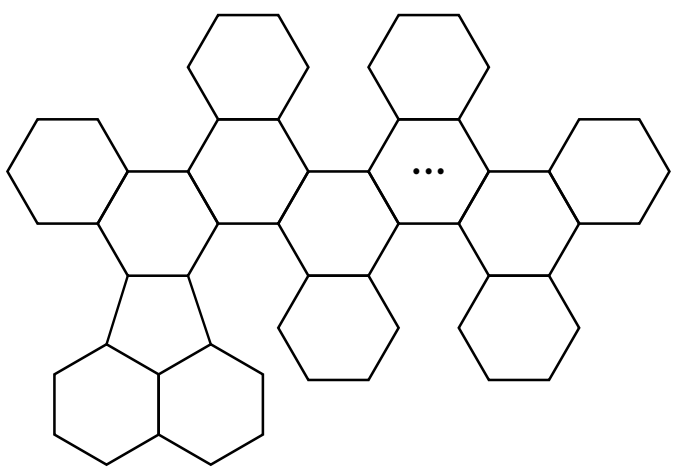

When $h$ is odd

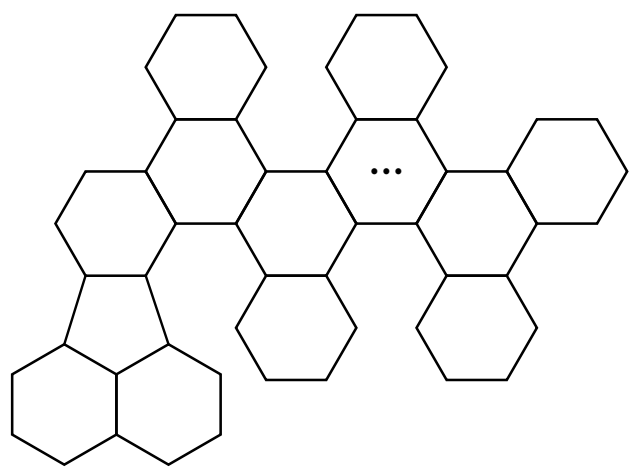

When $h>5$ is even

Figure 9: The fluoranthene system referred in Theorem 4.39.

Theorem 4.51 is similar to the right inequality of the eleventh part of Corollary 9 of the paper [93]. Additional extremal results concerning the minimum $A B C$ index of fluoranthene systems can be found from the general results obtained in [93].

\section{Open problems}

We end this paper by stating the following open problems regarding the $A B C$ index:

Characterize graphs attaining the

- minimum/maximum $A B C$ index from the class of all connected (i) graphs with a fixed size (ii) chemical cyclic graphs of a fixed order and fixed size.

- minimum $A B C$ index from the class of all trees of a fixed order and with a fixed (i) maximum degree (greater than 4) (ii) diameter (iii) number of pendent vertices (iv) perfect matching (v) number of branching vertices (the maximal version of this problem is also open when the number of branching vertices is greater than 2 (see Theorem 4.9)).

- minimum $A B C$ index among all connected cyclic graphs with a fixed order, size and with a fixed (i) maximum degree (ii) number of pendent vertices (iii) girth.

- minimum $A B C$ index among all connected graphs of a fixed order and with a fixed (i) independence number (ii) vertexconnectivity (iii) edge-connectivity (iv) number of pendent vertices (iv) chromatic number (v) minimum degree (vi) maximum degree.

\section{Acknowledgments}

This work is supported by the Serbian Ministry of Education, Science and Technological Development (Agreement No. 451-03-9/2021-14/200122) and by the bilateral cooperation between Serbia and Slovenia (Project No. BI-RS/20-21-025), and by the Scientific Research Deanship, University of Håil, Haail, Saudi Arabia (Project No. RG-20 031). 


\section{References}

[1] M. B. Ahmadi, D. Dimitrov, I. Gutman, S. A. Hosseini, Disproving a conjecture on trees with minimal atom-bond connectivity index, MATCH Commun. Math. Comput. Chem. 72 (2014) 685-698.

[2] I. R. Abdolhosseinzadeh, F. Rahbarnia, M. Tavakoli, Some vertex-degree-based topological indices under edge corona product, Ital. J. Pure Appl. Math. 38 (2017) 81-91.

[3] A. Ali, A. A. Bhatti, Extremal triangular chain graphs for bond incident degree (BID) indices, Ars Combin. 141 (2018) $213-227$.

[4] A. Ali, A. A. Bhatti, Z. Raza, Further inequalities between vertex-degree-based topological indices, Int. J. Appl. Comput. Math. 3 (2017) 19211930.

[5] A. Ali, A. A. Bhatti, Z. Raza, Topological study of tree-like polyphenylene systems, spiro hexagonal systems and polyphenylene dendrimer nanostars, Quantum Matter 5 (2016) 534-538.

[6] A. Ali, Z. Du, On the difference between atom-bond connectivity index and Randić index of binary and chemical trees Int. J. Quantum Chem. 117 (2017) Art\# e25446.

[7] A. Ali, Z. Raza, A. A. Bhatti, Bond incident degree (BID) indices of polyomino chains: a unified approach, Appl. Math. Comput. 287-288 (2016) $28-37$.

[8] A. Ali, Z. Raza, A. A. Bhatti, Extremal pentagonal chains with respect to bond incident degree indices, Canad. J. Chem. 94 (2016) 870-876.

[9] A. R. Ashrafi, T. Dehghan-Zadeh, N. Habibi, Extremal atom-bond connectivity index of cactus graphs, Commun. Korean Math. Soc. 30 (2015) 283-295.

[10] A. R. Ashrafi, T. Dehghan-Zadeh, N. Habibi, P. E. John, Maximum values of atom-bond connectivity index in the class of tricyclic graphs, J. Appl. Math. Comput. 50 (2016) 511-527.

[11] L. Berrocal , A. Olivieri, J. Rada, Extremal values of VDB topological indices over hexagonal systems with fixed number of vertices, Appl. Math. Comput. 243 (2014) 176-183.

[12] C. Betancur, R. Cruz, J. Rada, Vertex-degree-based topological indices over starlike trees, Discrete Appl. Math. 185 (2015) $18-25$.

[13] M. Bianchi, A. Cornaro, J. L. Palacios, A. Torriero, New upper bounds for the ABC index, MATCH Commun. Math. Comput. Chem. 76 (2016) $117-130$.

[14] T. Bıyıkoğlu, J. Leydold, Graphs with given degree sequence and maximal spectral radius, Electron. J. Combin. 15 (2008) Art\# R119.

[15] D. Bonchev, Overall connectivity - a next generation molecular connectivity, J. Mol. Graphics Model. 20 (2001) 65-75.

[16] J. A. Bondy, U. S. R. Murty, Graph Theory, Springer, London, 2008.

[17] G. Chartrand, L. Lesniak, P. Zhang, Graphs \& Digraphs, Sixth Edition, CRC Press, Boca Raton, 2016.

[18] X. Chen, On $A B C$ eigenvalues and $A B C$ energy, Linear Algebra Appl. 544 (2018) 141-157.

[19] X. Chen, K. C. Das, Solution to a conjecture on the maximum ABC index of graphs with given chromatic number, Discrete Appl. Math. 251 (2018) $126-134$.

[20] X. Chen, G. Hao, Extremal graphs with respect to generalized ABC index, Discrete Appl. Math. 243 (2018) 115-124.

[21] J. Chen, X. Guo, Extreme atom-bond connectivity index of graphs, MATCH Commun. Math. Comput. Chem. 65 (2011) 713-722.

[22] J. Chen, X. Guo, The atom-bond connectivity index of chemical bicyclic graphs, Appl. Math. J. Chinese Univ. 27 (2012) $243-252$.

[23] J. Chen, J. Liu, On atom-bond connectivity index of bicyclic graphs, J. Guangxi Teach. Educ. Univ. 28 (2011) 8-12.

[24] J. Chen, J. Liu, X. Guo, Some upper bounds for the atom-bond connectivity index of graphs, Appl. Math. Lett. 25 (2012) $1077-1081$.

[25] J. Chen, J. Liu, X. Guo, The atom-bond connectivity index of chemical unicyclic graphs, J. Zhejiang Univ. (Sci. Ed.) 39 (2012) 377-380.

[26] J. Chen, J. Liu, Q. Li, The atom-bond connectivity index of catacondensed polyomino graphs, Discrete Dyn. Nat. Soc. 2013 (2013) Art\# 598517.

[27] Z. Chen, J. Meng, Y. Tian, On atom-bond connectivity index of line and total graphs, Oper. Res. Trans. 17 (2013) 1-10.

[28] Y. H. Chen, H. Wang, X. D. Zhang, Note on extremal graphs with given matching number, Appl. Math. Comput. 308 (2017) $149-156$.

[29] R. Cruz, F. Duque, J. Rada, Extremal values of the number of inlets and number of bay regions over pericondensed hexagonal systems, $M A T C H$ Commun. Math. Comput. Chem. 78 (2017) 469-486.

[30] R. Cruz, H. Giraldo, J. Rada, Extremal values of vertex-degree topological indices over hexagonal systems, MATCH Commun. Math. Comput. Chem. 70 (2013) 501-512.

[31] R. Cruz, I. Gutman, J. Rada, Convex hexagonal systems and their topological indices, MATCH Commun. Math. Comput. Chem. 68 (2012) 97-108.

[32] R. Cruz, I. Gutman, J. Rada, Topological indices of Kragujevac trees, Proyecciones J. Math. 33 (2014) 471-482.

[33] R. Cruz, C. A. Marín, J. Rada, Vertex-degree-based topological indices over trees with two branching vertices Kragujevac J. Math. 43 (2019) 399-411.

[34] R. Cruz, J. Monsalve, J. Rada, On chemical trees that maximize atom-bond connectivity index, its exponential version, and minimize exponential geometric-arithmetic index, MATCH Commun. Math. Comput. Chem. 84 (2020) 691-718.

[35] R. Cruz, T. Pérez, J. Rada, Extremal values of vertex-degree-based topological indices over graphs, J. Appl. Math. Comput. 48 (2015) $395-406$.

[36] R. Cruz, J. Rada, Extremal polyomino chains of VDB topological indices, Appl. Math. Sci. 9 (2015) 5371-5388.

[37] Q. Cui, Q. Qian, L. Zhong, The maximum atom-bond connectivity index for graphs with edge-connectivity one, Discrete Appl. Math. 220 (2017) $170-173$.

[38] K. C. Das, Atom-bond connectivity index of graphs, Discrete Appl. Math. 158 (2010) 1181-1188.

[39] K. C. Das, S. Elumalai, I. Gutman, On ABC index of graphs, MATCH Commun. Math. Comput. Chem. 78 (2017) $459-468$.

[40] K. C. Das, I. Gutman, B. Furtula, On atom-bond connectivity index, Chem. Phys. Lett. 511 (2011) 452-454.

[41] K. C. Das, I. Gutman, B. Furtula, On atom-bond connectivity index, Filomat 26 (2012) 733-738.

[42] K. C. Das, A. D. Maden, I. N. Cangül, A. S. Çevik, On average eccentricity of graphs, Proc. Nat. Acad. Sci. India Sect. A 87 (2017) $23-30$.

[43] K. C. Das, M. A. Mohammed, I. Gutman, K. A. Atan, Comparison between atom-bond connectivity indices of graphs, MATCH Commun. Math. Comput. Chem. $\mathbf{7 6}$ (2016) 159-170.

[44] K. C. Das, J. M. Rodríguez, J. M. Sigarreta, On the maximal general ABC index of graphs with given maximum degree, Appl. Math. Comput. 386 (2020) Art\# 125531

[45] K. C. Das, N. Trinajstić, Comparison between first geometric-arithmetic index and atom-bond connectivity index, Chem. Phys. Lett. 497 (2010) $149-151$.

[46] T. Dehghan-Zadeh, A. R. Ashrafi, Atom-bond connectivity index of quasi-tree graphs, Rend. Circ. Mat. Palermo 63 (2014) $347-354$.

[47] T. Dehghan-Zadeh, A. R. Ashrafi, N. Habibi, Maximum values of atom-bond connectivity index in the class of tetracyclic graphs, J. Appl. Math. Comput. 46 (2014) 285-303.

[48] M. Dehmer, M. Grabner, B. Furtula, Structural discrimination of networks by using distance, degree and eigenvalue-based measures, $P L o S$ ONE 7 (2012) Art\# e38564.

[49] H. Deng, G. Huang, X. Jiang, A unified linear-programming modeling of some topological indices, J. Comb. Optim. 30 (2015) $826-837$.

[50] H. Deng, J. Yang, F. Xia, A general modeling of some vertex-degree based topological indices in benzenoid systems and phenylenes, Comput. Math. Appl. 61 (2011) 3017-3023.

[51] D. Dimitrov, Efficient computation of trees with minimal atom-bond connectivity index, Appl. Math. Comput. 224 (2013) 663-670.

[52] D. Dimitrov, On structural properties of trees with minimal atom-bond connectivity index, Discrete Appl. Math. 172 (2014) $28-44$.

[53] D. Dimitrov, On structural properties of trees with minimal atom-bond connectivity index II: Bounds on $B_{1}$ - and $B_{2}$-branches, Discrete Appl. Math. 204 (2016) 90-116.

[54] D. Dimitrov, On structural properties of trees with minimal atom-bond connectivity index IV: Solving a conjecture about the pendent paths of length three, Appl. Math. Comput. 313 (2017) 418-430. 
[55] D. Dimitrov, Extremal trees with respect to the atom-bond connectivity index, In: I. Gutman, B. Furtula, K. C Das, E. Milovanović, I. Milovanović (Eds.), Bounds in Chemical Graph Theory - Mainstreams, Mathematical Chemistry Monographs No. 20, Univ. Kragujevac, Kragujevac, 2017, pp. $53-67$.

[56] D. Dimitrov, Z. Du, A solution of the conjecture about big vertices of minimal-ABC trees, Appl. Math. Comput. 397 (2021) Art\# 125818.

[57] D. Dimitrov, Z. Du, Complete characterization of the minimal-ABC trees, Submitted.

[58] D. Dimitrov, Z. Du, C. M. da Fonseca, The minimal-ABC trees with $B_{1}$-branches, PLoS One 13 (2018) Art\# e0195153.

[59] D. Dimitrov, Z. Du, C. M. da Fonseca, On structural properties of trees with minimal atom-bond connectivity index III: Trees with pendent paths of length three, Appl. Math. Comput. 282 (2016) 276-290.

[60] D. Dimitrov, Z. Du, C. M. da Fonseca, Some forbidden combinations of branches in minimal-ABC trees, Discrete Appl. Math. 236 (2018) 165-182.

[61] D. Dimitrov, Z. Du, C. M. da Fonseca, The minimal-ABC trees with $B_{1}$-branches, PLoS ONE 13 (2018) e0195153.

[62] D. Dimitrov, B. Ikica, R. Škrekovski, Remarks on maximum atom-bond connectivity index with given graph parameters, Discrete Appl. Math. 222 (2017) $222-226$.

[63] D. Dimitrov, B. Ikica, R. Škrekovski, Remarks on the Graovac-Ghorbani index of bipartite graphs, Appl. Math. Comput. 293 (2017) 370-376.

[64] D. Dimitrov, N. Milosavljević, Efficient computation of trees with minimal atom-bond connectivity index revisited, MATCH Commun. Math. Comput. Chem. 79 (2018) 431-450.

[65] H. Dong, X. Wu, On the atom-bond connectivity index of cacti, Filomat 28 (2014) 1711-1717.

[66] Z. Du, On the atom-bond connectivity index and radius of connected graphs, J. Inequal. Appl. 2015 (2015) Art\# 188.

[67] Z. Du, C. M. da Fonseca, On a family of trees with minimal atom-bond connectivity, Discrete Appl. Math. 202 (2016) 37-49.

[68] Z. Du, D. Dimitrov, The minimal- $A B C$ trees with $B_{2}$-branches, Comput. Appl. Math. 39 (2020) Art\# 85.

[69] Z. Du, D. Dimitrov, The minimal- $A B C$ trees with $B_{1}$-branches II, IEEE Access 6 (2018) 66350-66366.

[70] Z. Dvořák, B. Lidický, R. Škrekovski, Randić index and the diameter of a graph, European J. Combin. 32 (2011) $434-442$.

[71] E. Estrada, Atom-bond connectivity and the energetic of branched alkanes, Chem. Phys. Lett. 463 (2008) $422-425$.

[72] E. Estrada, The ABC matrix, J. Math. Chem. 55 (2017) 1021-1033.

[73] E. Estrada, L. Torres, L. Rodríguez, I. Gutman, An atom-bond connectivity index: modelling the enthalpy of formation of alkanes, Indian J. Chem. Sec. A 37 (1998) 849-855.

[74] S. Fajtlowicz, On conjectures of Graffiti-II, Congr. Numer. 60 (1987) 187-197.

[75] G. A. Fath-Tabar, B. Vaez-Zadah, A. R. Ashrafi, A. Graovac, Some inequalities for the atom-bond connectivity index of graph operations, Discrete Appl. Math. 159 (2011) 1323-1330.

[76] B. Furtula, K. C. Das, I. Gutman, Comparative analysis of symmetric division deg index as potentially useful molecular descriptor, Int. J. Quantum Chem. 118 (2018) Art\# e25659.

[77] B. Furtula, A. Graovac, D. Vukičević, Atom-bond connectivity index of trees, Discrete Appl. Math. 157 (2009) $2828-2835$.

[78] B. Furtula, A. Graovac, D. Vukičević, Augmented Zagreb index, J. Math. Chem. 48 (2010) 370-380.

[79] B. Furtula, I. Gutman, K. C. Das, On atom-bond connectivity molecular structure descriptors, J. Serb. Chem. Soc. 81 (2016) 271-276.

[80] L. Gan, H. Hou, B. Liu, Some results on atom-bond connectivity index of graphs, MATCH Commun. Math. Comput. Chem. 66 (2011) 669-680.

[81] L. Gan, B. Liu, Z. You, The ABC index of trees with given degree sequence, MATCH Commun. Math. Comput. Chem. 68 (2012) 137-145.

[82] Y. Gao, Y. Shao, The smallest $A B C$ index of trees with $n$ pendent vertices, MATCH Commun. Math. Comput. Chem. 76 (2016) 141-158.

[83] M. Goubko, I. Gutman, Degree-based topological indices: Optimal trees with given number of pendents, Appl. Math. Comput. 240 (2014) $387-398$.

[84] M. Goubko, C. Magnant, P. Salehi Nowbandegani, I. Gutman, ABC index of trees with fixed number of leaves, MATCH Commun. Math. Comput. Chem. 74 (2015) 697-702.

[85] I. Gutman, Degree-based topological indices, Croat. Chem. Acta 86 (2013) 351-361.

[86] I. Gutman, B. Furtula (Eds.), Recent Results in the Theory of Randić Index, Univ. Kragujevac, Kragujevac, 2006.

[87] I. Gutman, B. Furtula, M. B. Ahmadi, S. A. Hosseini, P. Salehi Nowbandegani, M. Zarrinderakht, The ABC index conundrum, Filomat 27 (2013) $1075-1083$.

[88] I. Gutman, B. Ruščić, N. Trinajstić, C. F. Wilcox, Graph theory and molecular orbitals. XII. Acyclic polyenes, J. Chem. Phys. 62 (1975) $3399-3405$.

[89] I. Gutman, J. Tošović, S. Radenković, S. Marković, On atom-bond connectivity index and its chemical applicability, Indian J. Chem. Sec. A 51 (2012) 690-694.

[90] I. Gutman, N. Trinajstić, Graph theory and molecular orbitals. Total $\pi$-electron energy of alternant hydrocarbons, Chem. Phys. Lett. 17 (1972) $535-538$.

[91] I. Gutman, L. Zhong, K. Xu, Relating the $A B C$ and harmonic indices, J. Serb. Chem. Soc. 79 (2014) 557-563.

[92] F. Hayat, On generalized atom-bond connectivity index of cacti, Iranian J. Math. Chem. 10 (2019) 319-330.

[93] S. He, H. Chen, H. Deng, The vertex-degree-based topological indices of fluoranthene-type benzenoid systems, MATCH Commun. Math. Comput. Chem. 78 (2017) 431-458.

[94] M. Hemmasi, A. Iranmanesh, Some bounds on the modified Randić index, Kragujevac J. Sci. 37 (2015) 79-90.

[95] M. Hemmasi, A. Iranmanesh, Some inequalities for the atom-bond connectivity index of graph, J. Comput. Theor. Nanosci. 12 (2015) $2172-2179$.

[96] B. Hollas, The covariance of topological indices that depend on the degree of a vertex, MATCH Commun. Math. Comput. Chem. 54 (2005) 177-187.

[97] B. Horoldagva, I. Gutman, On some vertex-degree-based graph invariants, MATCH Commun. Math. Comput. Chem. 65 (2011) 723-730.

[98] S. A. Hosseini, M. B. Ahmadi, I. Gutman, Kragujevac trees with minimal atom-bond connectivity index, MATCH Commun. Math. Comput. Chem. $\mathbf{7 1}(2014) 5-20$.

[99] S. A. Hosseini, B. Mohar, M. B. Ahmadi, The evolution of the structure of $A B C$-minimal trees, arXiv:1804.02098v2 [math.CO] (2019).

[100] H. Hua, K. C. Das, H. Wang, On atom-bond connectivity index of graphs, J. Math. Anal. Appl. 479 (2019) 1099-1114.

[101] Y. Huang, Trees with given diameter minimizing the augmented Zagreb index and maximizing the ABC index, Commun. Math. Res. 33 (2017) $8-18$.

[102] X. Ke, Atom-bond connectivity index of benzenoid systems and fluoranthene congeners, Polycycl. Aromat. Comp. 32 (2012) 27-35.

[103] L. B. Kier, L. H. Hall, Molecular Connectivity in Structure-Activity Analysis, Wiley, New York, 1986.

[104] J. Li, On the ABC index of cacti, Int. J. Graph Theory Appl. 1 (2015) 57-66.

[105] X. Li, I. Gutman, Mathematical Aspects of Randić-Type Molecular Structure Descriptors, Univ. Kragujevac, Kragujevac, 2006.

[106] J. Li, J. B. Lv, Y. Liu, The harmonic index of some graphs, Bull. Malays. Math. Sci. Soc. 39 (2016) S331-S340.

[107] X. Li, Y. Shi, A survey on the Randić index, MATCH Commun. Math. Comput. Chem. 59 (2008) 127-156.

[108] J. Li, B. Zhou, Atom-bond connectivity index of unicyclic graphs with perfect matchings, Ars Combin. 109 (2013) 321-326.

[109] W. Lin, J. Chen, C. Ma, Y. Zhang, J. Chen, D. Zhang, J. Fei, On trees with minimal ABC index among trees with given number of leaves, MATCH Commun. Math. Comput. Chem. 76 (2016) 131-140.

[110] W. Lin, T. Gao, Q. Chen, X. Lin, On the atom-bond connectivity index of connected graphs with a given degree sequence, MATCH Commun. Math. Comput. Chem. 69 (2013) 571-578.

[111] W. Lin, P. Li, J. Chen, C. Ma, Y. Zhang, D. Zhang, On the minimal ABC index of trees with $k$ leaves, Discrete Appl. Math 217 (2017) $622-627$.

[112] W. Lin, J. Chen, Q. Chen, T. Gao, X. Lin, B. Cai, Fast computer search for trees with minimal ABC index based on tree degree sequences, MATCH Commun. Math. Comput. Chem. 72 (2014) 699-708.

[113] W. Lin, J. Chen, Z. Wu, D. Dimitrov, L. Huang, Computer search for large trees with minimal ABC index, Appl. Math. Comput. 338 (2018) $221-2308$.

[114] W. Lin, C. Ma, Q. Chen, J. Chen, T. Gao, B. Cai, Parallel search trees with minimal ABC index with MPI + OpenMP, MATCH Commun. Math. Comput. Chem. 73 (2015) 337-343.

[115] W. Lin, D. Dimitrov, R. Škrekovski, Complete characterization of trees with maximal augmented Zagreb Index, MATCH Commun. Math. Comput. Chem. 83 (2020) 167-178. 
[116] M. Liu, K. Xu, X. D. Zhang, Extremal graphs for vertex-degree-based invariants with given degree sequences, Discrete Appl. Math. 255 (2019) $267-277$.

[117] J . Liu, R . Zheng, J . Chen, B. Liu, The extremal general atom-bond connectivity indices of unicyclic and bicyclic graphs, MATCH Commun. Math. Comput. Chem. 81 (2019) 345-360.

[118] C. Magnant, P. S. Nowbandegani, I. Gutman, Which tree has the smallest ABC index among trees with $k$ leaves? Discrete Appl. Math. 194 (2015) $143-146$.

[119] B. Mohar, The structure of ABC-minimal trees with given number of leaves, MATCH Commun. Math. Comput. Chem. 79 (2018) 415-430.

[120] S. Nikolić, G. Kovačević, A. Miličević, N. Trinajstić, The Zagreb indices 30 years after, Croat. Chem. Acta 76 (2003) 113-124.

[121] J. L. Palacios, A resistive upper bound for the ABC index, MATCH Commun. Math. Comput. Chem. 72 (2014) $709-713$.

[122] J. L. Palacios, Bounds for the augmented Zagreb and the atom-bond connectivity indices, Appl. Math. Comput. 307 (2017) 141-145.

[123] J. L. Palacios, Correction to "Bounds for the augmented Zagreb and the atom-bond connectivity indices", Appl. Math. Comput. 362 (2019) Art\# 124559.

[124] K. Pattabiraman, On topological indices of graph transformation, Int. J. Appl. Comput. Math. 3 (2017) 1171-1184.

[125] J. Rada, R. Cruz, I. Gutman, Vertex-degree-based topological indices of catacondensed hexagonal systems, Chem. Phys. Lett. 572 (2013) $154-157$.

[126] M. Rakić, B. Furtula, A novel method for measuring the structure sensitivity of molecular descriptors, J. Chemom. 33 (2019) Art\# e3138.

[127] M. Randić, On characterization of molecular branching, J. Amer. Chem. Soc. 97 (1975) 6609-6615.

[128] M. Randić, On history of the Randić index and emerging hostility toward chemical graph theory, MATCH Commun. Math. Comput. Chem. 59 (2008) $5-124$

[129] M. Randić, The connectivity index 25 years after, J. Mol. Graph. Model. 20 (2001) 19-35.

[130] Y. Rao, A. Aslam, M. U. Noor, A. O. Almatroud, Z. Shao, Bond incident degree indices of catacondensed pentagonal systems, Complexity 2020 (2020) Art\# 4935760

[131] Z. Raza, A. A. Bhatti, A. Ali, More on comparison between first geometric-arithmetic index and atom-bond connectivity index, Miskolc Math. Notes 17 (2016) 561-570.

[132] Z. Shao, P. Wu, Y. Gao, I. Gutman, X. Zhang, On the maximum ABC index of graphs without pendent vertices, Appl. Math. Comput. 315 (2017) 298-312.

[133] Z. Shao, P. Wu, H. Jiang, S. M. Sheikholeslami, S. Wang, On the maximum ABC index of bipartite graphs without pendent vertices, Open Chem. 18 (2020) 39-49.

[134] Z. Shao, P. Wu, X. Zhang, D. Dimitrov, J. Liu, On the maximum $A B C$ index of graphs with prescribed size and without pendent vertices, IEEE Access 6 (2018) 27604-27616.

[135] R. Škrekovski, D. Dimitrov, J. Zhong, H. Wu, W. Gao, Remarks on multiplicative atom-bond connectivity index, IEEE Access 7 (2019) $76806-76811$.

[136] X. Tan, M. Liu, J. Liu, On the maximal general ABC index of graphs with fixed maximum degree, MATCH Commun. Math. Comput. Chem. 85 (2021) 121-130.

[137] N. Trinajstić, Chemical Graph Theory, CRC Press, Boca Raton, 1992.

[138] D. Vukičević, J. Đurdević, Bond additive modeling 10. Upper and lower bounds of bond incident degree indices of catacondensed fluoranthenes, Chem. Phys. Lett. 515 (2011) 186-189.

[139] D. Vukičević, B. Furtula, Topological index based on the ratios of geometrical and arithmetical means of end-vertex degrees of edges, J. Math. Chem. 46 (2009) 1369-1376.

[140] D. Vukičević, M. Gašperov, Bond additive modeling 1. Adriatic indices, Croat. Chem. Acta 83 (2010) 243-260.

[141] L. Wu, Y. Hu, Atom-bond connectivity index and diameter of graphs, J. Hebei Univ. Sci. Tech. 37 (2016) 552-555.

[142] X. Wu, L. Zhang, On structural properties of ABC-minimal chemical trees, Appl. Math. Comput. 362 (2019) Art\# 124570.

[143] R. Xing, B. Zhou, Extremal trees with fixed degree sequence for atom-bond connectivity index, Filomat 26 (2012) 683-688.

[144] R. Xing, B. Zhou, F. Dong, On atom-bond connectivity index of connected graphs, Discrete Appl. Math. 159 (2011) 1617-1630.

[145] R. Xing, B. Zhou, Z. Du, Further results on atom-bond connectivity index of trees, Discrete Appl. Math. 158 (2010) 1536-1545.

[146] X. D. Zhang, The Laplacian spectral radii of trees with degree sequence, Discrete Math. 308 (2008) 3143-3150.

[147] G. Zhang, Y. Chen, Functions on adjacent vertex degrees of graphs with prescribed degree sequence, MATCH Commun. Math. Comput. Chem. 80 (2018) 129-139.

[148] X. M. Zhang, Y. Sun, H. Wang, X. D. Zhang, On the ABC index of connected graphs with given degree sequences, J. Math. Chem. 56 (2018) $568-582$.

[149] X. M. Zhang, Y. Yang, H. Wang, X. D. Zhang, Maximum atom-bond connectivity index with given graph parameters, Discrete Appl. Math. 215 (2016) 208-217.

[150] X. M. Zhang, X. D. Zhang, R. Bass, H. Wang, Extremal trees with respect to functions on adjacent vertex degrees, MATCH Commun. Math. Comput. Chem. 78 (2017) 307-322.

[151] Y. Zheng, W. Lin, Q. Chen, L. Huang, Z. Wu, Characterizing trees with minimal $A B C$ index with computer search: a short survey, Open J. Discrete Appl. Math 1 (2018) 1-9.

[152] L. Zhong, Q. Cui, On a relation between the atom-bond connectivity and the first geometric-arithmetic indices, Discrete Appl. Math. 185 (2015) 249-253.

[153] L. Zhong, K. Xu, Inequalities between vertex-degree-based topological Indices, MATCH Commun. Math. Comput. Chem. 71 (2014) 627-642.

[154] B. Zhou, N. Trinajstić, On a novel connectivity index, J. Math. Chem. 46 (2009) 1252-1270.

[155] B. Zhou, R. Xing, On atom-bond connectivity index, Z. Naturforsch. 66 (2011) 61-66.

[156] W. N. Zuki, Z. Du, M. K. Jamil, R. Hasni, Extremal trees with respect to the difference between atom-bond connectivity index and Randić index, Symmetry 12 (2020) Art\# 1591. 